\title{
Bathyal cumacean assemblages from the southern margin of the Cap Ferret Canyon (SE Bay of Biscay)
}

\author{
Jordi Corbera ${ }^{1}$, Jean Claude Sorbe ${ }^{2 \dagger}$ \\ ${ }^{1}$ Carrer Gran 90, 08310 Argentona, Catalonia, Spain. \\ (JC) (Corresponding author) E-mail: corberajordi@gmail.com. ORCiD ID: https://orcid.org/0000-0003-3583-3929 \\ ${ }^{2}$ Muséum national d'Histoire naturelle, Institut de Systématique, Évolution, Biodiversité IS YEB - UMR 7205 - CNRS, \\ MNHN, UPMC, EPHE, 57 rue Cuvier, CP 26, 75005 Paris, France. \\ $\dagger$ deceased on 30 December 2019
}

\begin{abstract}
Summary: The structure of the cumacean assemblages from the southern margin of the Cap Ferret Canyon was studied at 13 stations ranging from 346 to 1099 m depth with a modified Macer-GIROQ suprabenthic sledge (four superimposed nets; 0.5 $\mathrm{mm}$ mesh size). A total of 1885 specimens were collected and classified into 5 families and 42 species. The total abundances fluctuated between 2.8 ind./100 $\mathrm{m}^{2}$ (station TS04; 484-485 m) and 55.8 ind./100 $\mathrm{m}^{2}$ (station TS08; 714-708 m). The highest values of species richness and diversity were recorded at station TS13 (1097-1099 m): $\mathrm{S}=25$ species; $\mathrm{H}^{\prime}\left(\log _{2}\right)=4.05$. The near-bottom vertical distribution of the cumacean fauna showed the same pattern at all stations: at least $60 \%$ of the individuals were sampled by the lower net of the sledge and a drastic abundance decrease occurred between the two lowermost water layers sampled by the sledge. The multivariate analysis carried out on abundance data discriminated three main groups of stations distributed across depth (TS09 excluded): group Ia (346-485 m) characterized by the dominance of Nannastacidae $(57.2 \%)$ at family level and Campylaspis sulcata, Leptostylis macrura at species level; group Ib (522-714 m) characterized by the dominance of Nannastacidae $(66.1 \%)$ at family level and Campylaspis squamifera, C. laevigata and Leptostylis macrura at species level; and group II (790-1099 m) characterized by the dominance of Diastylidae (40.3\%) at family level and Makrokylindrus (Adiastylis) josephinae, Leucon (Epileucon) pusillus and Diastyloides serratus at species level. According to this analysis, the main faunal change occurs between group I and II between 714 and $790 \mathrm{~m}$, in relation to changes in the texture of surficial sediments. Although bathyal cumacean assemblages appear to be less abundant than those studied on continental shelves, they are significantly more diverse. However, such results could be in part related to the use of different
\end{abstract} sampling methods.

Keywords: Cumacea; assemblages; suprabenthos; bathyal; Bay of Biscay; NE Atlantic Ocean.

Comunidades batiales de cumáceos en el margen sur del cañón de Cap Ferret (SE del golfo de Vizcaya)

Resumen: Se ha estudiado la estructura de las comunidades de cumáceos del margen sur del cañón de Cap Ferret en 13 estaciones, distribuídas desde los 346 hasta los 1099 m de profundidad con un patín suprabentónico Macer-GIROQ modificado (cuatro redes superpuestas; $0.5 \mathrm{~mm}$ de malla). En total, se recolectaron 1885 especímenes que fueron clasificados en cinco familias y 42 especies. La densidad total varió entre 2.8 ind./100 m² (estación TS04; 484-485 m) y 55.8 ind./100 m² (estación TS08; 714-708 m). Los valores máximos de riqueza específica y diversidad se obtuvieron en la estación TS13 (1097-1099 m): $S=25$ especies; $H^{\prime}\left(\log _{2}\right)=4.05$. La distribución vertical cercana al fondo de la fauna de cumáceos mostró el mismo patrón en las diferentes estaciones: al menos el $60 \%$ de los individuos fue muestreado en la red inferior del patín y se observó un descenso drástico de la abundancia entre las dos capas de agua inferiores muestreadas por el patín. El análisis multivariante llevado a cabo con los datos de densidad discrimina tres grupos principales de estaciones (excluida TS09) distribuidos según la profundidad: el grupo Ia $(346-485 \mathrm{~m})$ caracterizado por la dominancia de los Nannastacidae $(57.2 \%)$ a nivel de familia, y por Campylaspis sulcata y Leptostylis macrura a nivel de especie; el grupo Ib (522-714 m) caracterizado también por la dominancia de los Nannastacidae $(66.1 \%)$ a nivel de familia, pero por Campylaspis squamifera, C. laevigata y Leptostylis macrura a nivel de especie; el grupo II (790-1099 m) caracterizado por la dominancia de los Diastylidae (40.3\%) a nivel de familia, y por Makrokylindrus (Adiastylis) josephinae, Leucon (Epileucon) pusillus y Diastyloides serratus a nivel de especie. De acuerdo con este análisis, el principal cambio faunístico ocurre entre los grupos I y II entre 714 y $790 \mathrm{~m}$, relacionado con cambios en la textura de los sedimentos superficiales. Aunque las comunidades de cumáceos batiales parecen ser menos densas que las estudiadas en la plataforma continental, son significativamente más diversas. No obstante, estos resultados podrían estar en parte relacionados con el uso de métodos de muestreo diferentes.

Palabras clave: Cumacea; comunidades; suprabentos; batial; golfo de Bizcaya; océano Atlántico NE.

Citation/Como citar este artículo: Corbera J., Sorbe J.C. 2020. Bathyal cumacean assemblages from the southern margin of the Cap Ferret Canyon (SE Bay of Biscay). Sci. Mar. 84(2): 167-179. https://doi.org/10.3989/scimar.05031.07A

Editor: J.A. Cuesta.

Received: January 9, 2020. Accepted: April 30, 2020. Published: May 11, 2020.

Copyright: (C) 2020 CSIC. This is an open-access article distributed under the terms of the Creative Commons Attribution 4.0 International (CC BY 4.0) License. 


\section{INTRODUCTION}

Cumaceans (more than 1750 known species; see Watling and Gerken 2019) are small peracarid crustaceans widely distributed from intertidal to hadal bottoms (Jones 1969) and showing the highest diversity in bathyal environments (Jones and Sanders 1972, Reyss 1973, Gage and Tyler 1992), a bathymetric distributional pattern also recognized for most benthic taxa (Rex et al. 1997, Rex and Etter 2010). Furthermore, they represent one of the main faunal components of marine suprabenthic assemblages (Mees and Jones 1997) because they live close to the sediment-water interface, burrowing in the top layer substratum or swimming in the near-bottom water (see Foxon 1936, Forsman 1938, Dixon 1944).

The deep cumacean fauna of the NE Atlantic Ocean (including the Bay of Biscay) is probably one of the best known in the world thanks to the taxonomical works of Bonnier (1896), Fage (1929), Reyss (1974a, b, 1978), Jones (1974, 1984, 1985) and Bishop (1981a, b) but also to the pioneering study of Lagardère (1977) on their bathymetric distribution in the southern part of the Bay of Biscay. However, quantitative information on the structure of the corresponding taxocœnoses remains very scarce in this area. To fill this gap, a new research programme was initiated in 1984 on the structure of bathyal suprabenthic communities from the southern margin of the Cap Ferret Canyon (SE Bay of Biscay, off Arcachon Bay). During the ESSAIS and ECOFER cruises carried out in 1989, a new set of stations located along a bathymetric gradient was sampled with a suprabenthic sledge. The abundant material collected with this gear was studied at the highest taxonomical level (Dauvin et al. 1995) as well as at the lowest one for all the suprabenthic fauna collected at three stations (Elizalde et al. 1993a, Elizalde 1994) and for mysids (Elizalde et al. 1991) and amphipods (Dauvin and Sorbe 1995) according to the species identification progress. According to Dauvin et al. (1995), cumaceans are the fourth most abundant group in this material $(5.6 \%$ of the total collected fauna), after isopods $(50.8 \%)$, amphipods $(22.7 \%)$ and mysids $(14.1 \%)$, and their percentage contribution increases with depth in the study area $(0.5 \%-22.4 \%$ of the whole fauna collected at each sampling station). The present work is a new contribution to the knowledge of bathyal suprabenthic assemblages from the Cap Ferret area aimed at describing patterns of bathymetric as well as the near-bottom vertical distribution of cumacean species, estimating abundance of individuals and characterizing inter-specific associations.

\section{MATERIALS AND METHODS}

\section{Study area}

The study area is located on bathyal soft bottoms from the southern margin of the Cap Ferret Canyon. In that area, surficial sediments were sampled during previous surveys with box corers for granulometric analyses and estimation of their organic content (see data in Elizalde et al. 1991, 1993b, Elizalde 1994). Etcheber et al. (1999) synthesized the whole available sedimentological information accumulated in the Cap Ferret region (canyon and its lateral margins). On the southern margin of the canyon, the bathymetric distribution of the sediment fractions is clearly related to the general morphology of the area, showing a narrow sandy lobe extending down to $800 \mathrm{~m}$ depth, to the north of the study area. This feature is probably related to the impact of alongslope bottom currents limiting the local deposition of fine particles (see Durrieu de Madron et al. 1999). In the study area, the mean grain size decreases with depth, from $160 \mu \mathrm{m}$ at the shelf break to a value stabilized around $10 \mu \mathrm{m}$ from about $600 \mathrm{~m}$ down to $3000 \mathrm{~m}$. Conversely, the carbon organic content of sediments (measured in the $1 \mathrm{~cm}$ top layer of cores, expressed as $\%$ of sediment dry weight) increases with depth, between $0.23 \%$ at the shelf break and $1.36 \%$ at around $1000 \mathrm{~m}$ depth. The dominant fractions ( $>50 \%$ ) allow two sedimentary zones to be distinguished according to depth: an upper muddy sand zone characterized by a decreasing dominance of fine sands with depth and a lower mud zone characterized by a dominance of fine silts and clay, and by stabilized values of the mean grain size according to depth. Following the definition given by Stanley et al. (1983), the mud-line is therefore located at about $600 \mathrm{~m}$ depth in the study area, a limit representing the erosion-deposition boundary beneath which the organic content of surficial sediments increases in response to the deposition of silty and/or clayey particles (Etcheber et al. 1999).

According to Durrieu de Madron et al. (1999), two superimposed water masses were detected during the ECOFER experiments in the upper part of the Cap Ferret region, inferred from CTD profiles within the Cap Ferret Canyon: (1) a low-salinity water mass extending between 200 and $600 \mathrm{~m}$ in the water column, referred to as Eastern North Atlantic Water and characterized by a salinity minimum of 35.51 and a temperature of $10.8^{\circ} \mathrm{C}$ at $500 \mathrm{~m}$ water depth; and (2) a high saline core of Mediterranean Overflow Water detected between 700 and $1300 \mathrm{~m}$ water depth, characterized a salinity maximum of 35.76 , a temperature of $9.8^{\circ} \mathrm{C}$ and an oxygen minimum of $3.6 \mathrm{ml} \mathrm{L}^{-1}$ at about $1000 \mathrm{~m}$ water depth. Furthermore, an intermediate nepheloid layer centred at a depth of around $500 \mathrm{~m}$ and horizontally detached from the seafloor was detected at the head and on the flanks of the canyon during all five ECOFER experiments (1989-1991). It is supposed that both Eastern North Atlantic Water and Mediterranean Overflow Water impinge on the slope in the study area (canyon southern margin) and therefore impact the structure of the underlying bathyal benthic communities.

\section{Sampling}

During the ESSAIS I, ESSAIS II and ECOFER I surveys carried out between April and July 1989, 13 stations ranging from 346 to $1099 \mathrm{~m}$ depth (Fig. 1; Table 1) were sampled with a modified Macer-GIROQ 
suprabenthic sledge (full description in Dauvin et al. 1995). This gear is equipped with four superimposed nets $(0.5 \mathrm{~mm}$ mesh size $)$ that simultaneously sample four water layers above the seafloor $(\mathrm{N} 1,10-40 \mathrm{~cm}$; $\mathrm{N} 2$, 45-75 cm; N3, 80-110 cm; N4, 115-145 cm) and with an opening-closing system of these nets acting by contact with the seafloor. Each net is fixed on a rectangular metallic box (height, $30 \mathrm{~cm}$; width, 60 $\mathrm{cm}$ ) equipped with a TSK flowmeter that estimates the haul length and the bottom area swept by the sledge during each haul (calculated from the mean value of the available flowmeter measurements; see Table 1). Based on these estimates, the standardized abundances of species are expressed in individuals $/ 100 \mathrm{~m}^{2}$ (cumulative values from the four sledge nets N1-N4). The sledge was towed over the seafloor at a speed of 1-2 knots. All samplings were carried out during daytime (between 8 and $18 \mathrm{~h}$ ), except TS06 and TS13, which were carried out at night before midnight (see Table 1). The collected material was fixed on board with a solution of $10 \%$ neutral formalin in sea water until sorting into major taxonomical groups at the laboratory. All groups (including cumaceans) were then transferred to and preserved in $70 \%$ ethanol until species identification.

\section{Data analyses}

Following Brunel (1972) and Sainte-Marie and Brunel (1985), an index K of swimming activity above the bottom was computed for each species, expressed as follows:

$$
\begin{aligned}
& \mathrm{K} 1=\sum \mathrm{N} 1 / \sum \mathrm{Nt} \\
& \mathrm{K} 2=\sum \mathrm{N} 2 / \sum \mathrm{Nt} \\
& \mathrm{K} 3=\sum \mathrm{N} 3 / \sum \mathrm{Nt} \\
& \mathrm{K} 4=\sum \mathrm{N} 4 / \sum \mathrm{Nt}
\end{aligned}
$$

where $\sum \mathrm{N} 1, \sum \mathrm{N} 2, \sum \mathrm{N} 3$ and $\sum \mathrm{N} 4$ are the abundances (number of individuals) in the 10-40, 45-75, 80-110 and $115-145 \mathrm{~cm}$ water layers above the bottom, respectively; and $\sum \mathrm{Nt}$ is the total number of individuals in the four water layers sampled by the sledge (cumulative values from all available water layers and stations, excluding TS09). This $\mathrm{K}$ index can vary

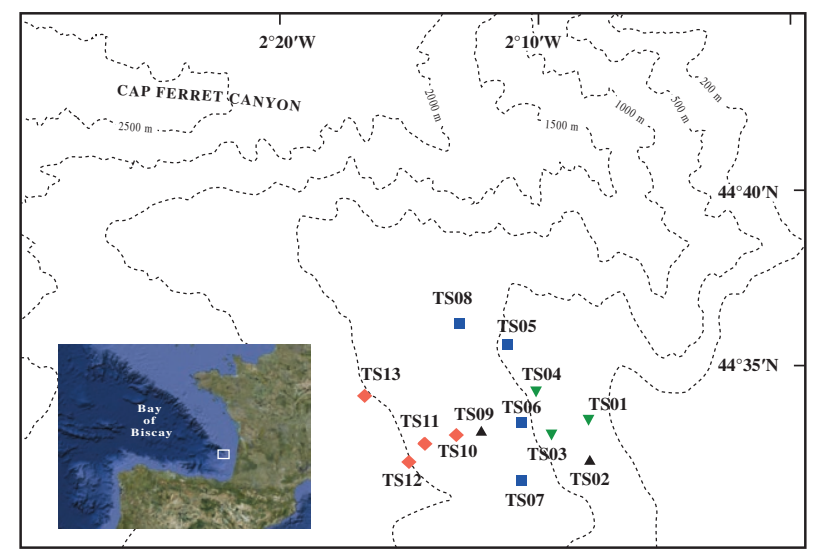

Fig. 1. - Geographical location of the 13 slope stations sampled with a suprabenthic sledge on the southern margin of the Cap Ferret Canyon (ESSAIS and ECOFER oceanographic cruises). Station symbols: green triangles, assemblage Ia; blue squares, assemblage Ib; red diamonds, assemblage II; black triangles, stations outside the groups or not analysed (see text).

from 0 (when a given species is absent at the level considered) to 1 (when all the specimens are sampled in the same water layer).

Abundance data (Dt, ind./100 $\mathrm{m}^{2}$ ) were analysed using the Plymouth Routine in Multivariate Ecological Research (PRIMER v 5.0) software package (Clarke and Gorley 2001). Univariate diversity indices (Shannon-Wiener diversity $\mathrm{H}^{\prime}$ using $\log _{2}$, Pielou evenness J') were calculated from species abundances with the DIVERSE routine. A matrix of similarity between samples was constructed by means of the Bray-Curtis measure applied to squareroot transformed species abundances in order to down-weight the contribution of abundant species. From this matrix, the 12 suprabenthic samples (sample TS09 excluded - damaged material) were classified by cluster analysis based on the complete linkage sorting algorithm. A graphical ordination was carried out on the same matrix using non-metric multidimensional scaling. Finally, the SIMPER routine was used to identify species that most contributed to within-group similarity and between-group dissimilarity.

Mean values of data sets were statistically compared using t tests according to Dagnelie (1975).

Table 1. - Haul characteristics and geographical position of stations sampled with a suprabenthic sledge on the southern margin of the Cap

\begin{tabular}{|c|c|c|c|c|c|c|c|}
\hline Cruise & Haul code & $\begin{array}{c}\text { Date } \\
(\mathrm{d} / \mathrm{m} / \mathrm{y})\end{array}$ & $\begin{array}{l}\text { Time }^{\mathrm{a}} \\
\text { (h:m) }\end{array}$ & $\mathrm{N}$ & W & $\begin{array}{c}\text { Depth }^{b} \\
\text { (m) }\end{array}$ & $\begin{array}{l}\text { Area } \\
\left(\mathrm{m}^{2}\right)\end{array}$ \\
\hline ESSAIS I & TS01 & $21 / 4 / 89$ & $15: 50$ & $44^{\circ} 33.30^{\prime}$ & $2^{\circ} 08.30^{\prime}$ & $346-347$ & 512 \\
\hline ESSAIS II & TSO2 & $18 / 5 / 89$ & $08: 11$ & $44^{\circ} 32.58^{\prime}$ & $2^{\circ} 08.17^{\prime}$ & $390-383$ & 171 \\
\hline ESSAIS II & TS03 & $18 / 5 / 89$ & $17: 59$ & $44^{\circ} 33.11^{\prime}$ & $2^{\circ} 09.85^{\prime}$ & $425-437$ & 384 \\
\hline ESSAIS II & TS04 & $18 / 5 / 89$ & $10: 43$ & $44^{\circ} 34.38^{\prime}$ & $2^{\circ} 10.18^{\prime}$ & $485-484$ & 443 \\
\hline ECOFER I & TS05 & $01 / 7 / 89$ & $13: 20$ & $44^{\circ} 35.57^{\prime}$ & $2^{\circ} 11.21^{\prime}$ & $523-522$ & 519 \\
\hline ESSAIS I & TS06 & $21 / 4 / 89$ & $22: 27$ & $44^{\circ} 33.40^{\prime}$ & $2^{\circ} 10.70^{\prime}$ & $608-611$ & 807 \\
\hline ESSAIS I & TS07 & $21 / 4 / 89$ & $17: 19$ & $44^{\circ} 31.90^{\prime}$ & $2^{\circ} 10.80^{\prime}$ & $660-714$ & 928 \\
\hline ECOFER I & TS08 & $01 / 7 / 89$ & $15: 59$ & $44^{\circ} 36.21^{\prime}$ & $2^{\circ} 12.84^{\prime}$ & $714-708$ & 375 \\
\hline ESSAIS II & TS09 & $18 / 5 / 89$ & $16: 31$ & $44^{\circ} 33.22^{\prime}$ & $2^{\circ} 12.48^{\prime}$ & $740-754$ & 370 \\
\hline ESSAIS II & TS10 & $18 / 5 / 89$ & $13: 36$ & $44^{\circ} 33.10^{\prime}$ & $2^{\circ} 13.13^{\prime}$ & $791-790$ & 328 \\
\hline ESSAIS II & TS11 & $18 / 5 / 89$ & $15: 00$ & $44^{\circ} 32.89^{\prime}$ & $2^{\circ} 14.24^{\prime}$ & $923-924$ & 279 \\
\hline ESSAIS I & TS12 & $22 / 4 / 89$ & $8: 33$ & $44^{\circ} 32.30^{\prime}$ & $2^{\circ} 15.10^{\prime}$ & 1024-1043 & 477 \\
\hline ESSAIS II & TS13 & $17 / 5 / 89$ & $23: 10$ & $44^{\circ} 34.19^{\prime}$ & $2^{\circ} 16.18^{\prime}$ & 1097-1099 & 382 \\
\hline
\end{tabular}
Ferret Canyon during the ESSAIS and ECOFER cruises. Estimated bottom area swept by the sledge during each haul (calculated from TSK flowmeter measurements). ${ }^{\mathrm{a}}$ : time and position of the boat at the beginning of the haul on the bottom; ${ }^{\mathrm{b}}$ : depth (below the boat) at the beginning and at the end of the haul on the bottom. 


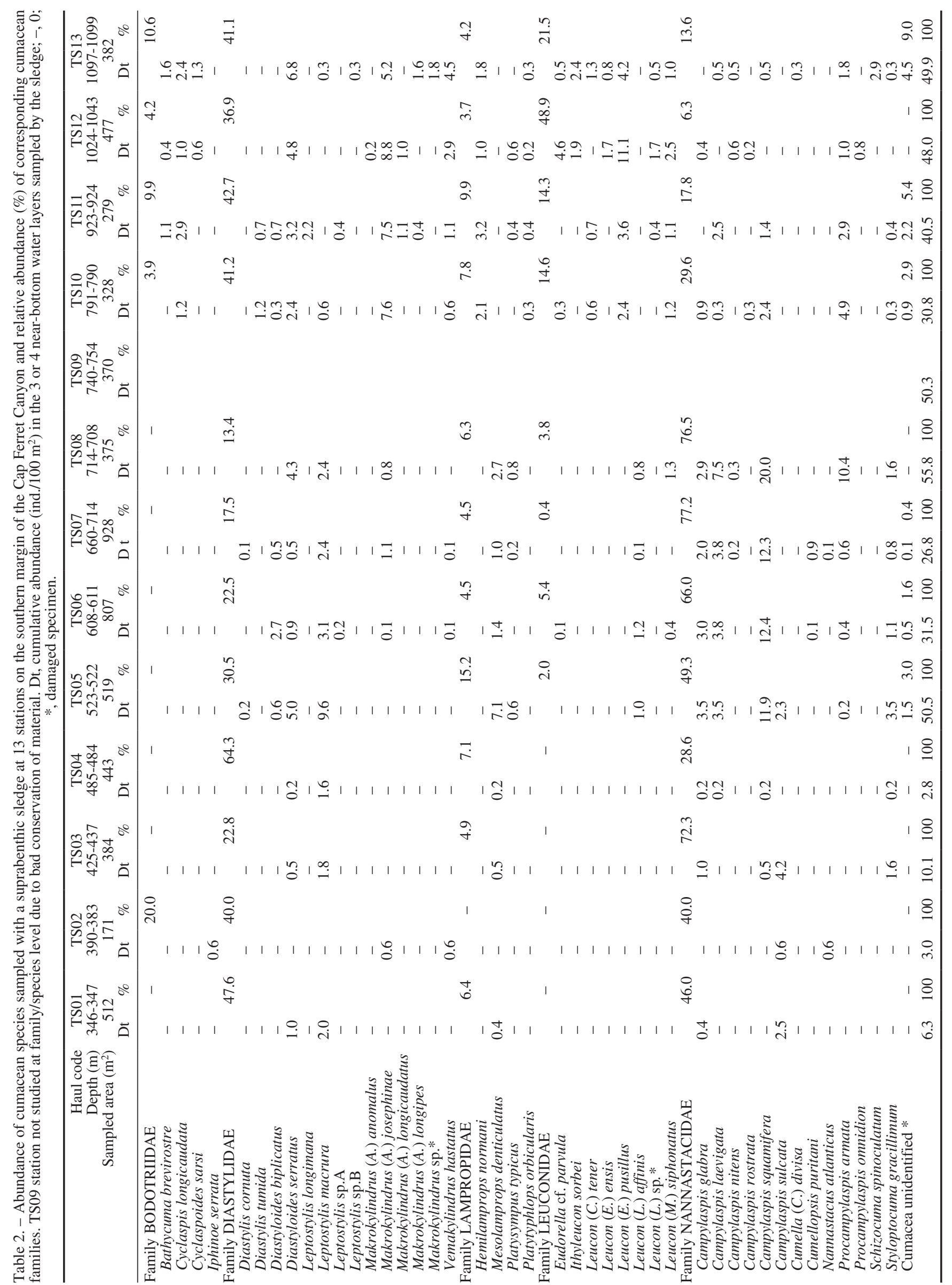


Table 3. - Species richness (S), total abundance (Dt, ind $/ 100 \mathrm{~m}^{2}$ ), Shannon-Wiener diversity index $\left(\mathrm{H}^{\prime}, \log _{2}\right)$ and Pielou evenness $\left(\mathrm{J}^{\prime}\right)$ of the cumacean fauna sampled with a suprabenthic sledge at 13 stations on the southern margin of the Cap Ferret Canyon (values from nets N1-N4). -, no data.

\begin{tabular}{lcccc}
\hline Haul code & S & Dt & H' & J' \\
\hline TS01 & 5 & 6.3 & 1.98 & 0.85 \\
TS02 & 5 & 3.0 & 2.32 & 1.00 \\
TS03 & 7 & 10.1 & 2.37 & 0.84 \\
TS04 & 7 & 2.8 & 2.09 & 0.75 \\
TS05 & 13 & 50.5 & 3.05 & 0.83 \\
TS06 & 16 & 31.5 & 2.88 & 0.72 \\
TS07 & 17 & 26.8 & 2.76 & 0.67 \\
TS08 & 13 & 55.8 & 2.86 & 0.77 \\
TS09 & - & 50.3 & - & - \\
TS10 & 19 & 30.8 & 3.53 & 0.83 \\
TS11 & 22 & 40.5 & 3.93 & 0.88 \\
TS12 & 22 & 48.0 & 3.60 & 0.81 \\
TS13 & 25 & 49.9 & 4.05 & 0.87 \\
\hline
\end{tabular}

\section{RESULTS}

A total of 1885 cumacean specimens were collected. Of these, $87.7 \%$ were identified and classified into 5 families (Nannastacidae, 47.5\%; Diastylidae, 30.1\%; Leuconidae, 12.7\%; Lampropidae, 6.9\%; Bodotriidae, $2.8 \%$ ) and 42 species (Table 2; Supplementary Material Table S1). The number of species (species richness) per station ranged from 5 at stations TS01 and TS02 to 25 at station TS13 (Table 3). The total abundances fluctuated between a minimum of 2.8 ind./100 $\mathrm{m}^{2}$ at station TS04 and a maximum of 55.8 ind./100 $\mathrm{m}^{2}$ at station TS08. The diversity indexes H' ranged between 1.98 (TS01) and 4.05 (TS13). All these three structural indices are significantly correlated with depth (species richness $\mathrm{r}=0.960, \mathrm{p}<0.001$; abundances $\mathrm{r}=0.732$, $\mathrm{p}<0.01$; diversity $\mathrm{r}=0.927, \mathrm{p}<0.001$ ). Even at station TS07 where the nannastacid Camplylaspis squamifera was highly dominant (45.9\% of the individuals), the evenness values, J', were relatively high $(\geq 0.67)$ but were correlated neither with depth $(\mathrm{r}=0.078, \mathrm{p}>0.05)$ nor with $\mathrm{H}^{\prime}$ values $(\mathrm{r}=0.093, \mathrm{p}>0.05)$.

At family level, samples from the $0-145 \mathrm{~cm}$ water layer were numerically dominated by Nannastacidae or Diastylidae in the shallower part of the study area

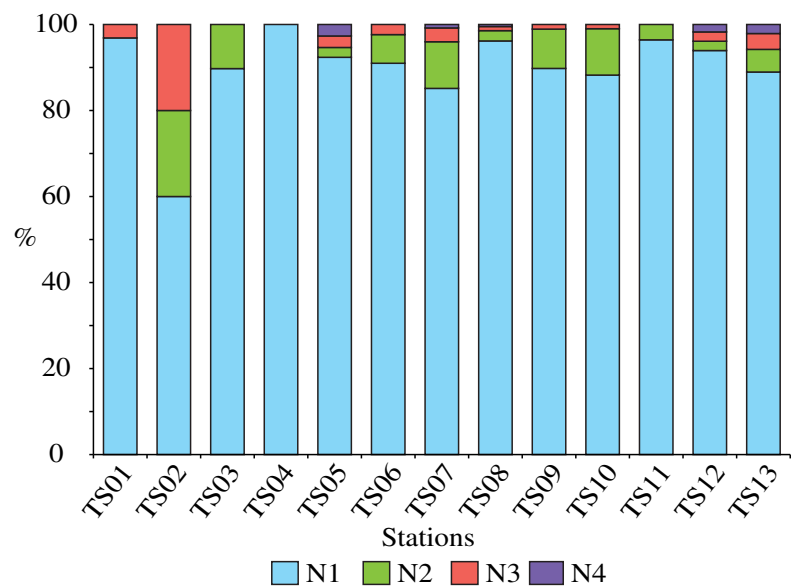

Fig. 2. - Percentage contribution of nets N1-N4 samples to the whole suprabenthic cumacean fauna sampled by the sledge at each of the 13 slope stations from the southern margin of the Cap Ferret Canyon. (although with low abundances between 346 and 485 $\mathrm{m})$, and the Nannastacidae were progressively replaced by Leuconidae below $700 \mathrm{~m}$ depth. At species level (Table 2), the highest abundances in the $0-145 \mathrm{~cm}$ water layer were recorded for the Nannastacidae Campylaspis squamifera (20.0 ind./100 $\mathrm{m}^{2}$, TS08), the Leuconidae Leucon (Epileucon) pusillus (11.1 ind./100 m², TS12) and the Nannastacidae Procampylaspis armata (10.4 ind./100 m², TS08). The Diastylidae Diastyloides serratus showed the widest bathymetric distribution in the study area, being captured between 346 and $1099 \mathrm{~m}$ depth (present at 11 stations).

As shown in Figure 2, the near-bottom vertical distribution of the cumacean fauna showed the same pattern at every station: at least $60.0 \%$ of the individuals were sampled by the lower net of the sledge, whereas only $0 \%$ to $2.7 \%$ of them were captured at the uppermost level, demonstrating that these small peracarids

Table 4. - Index (K) of swimming activity (see text) calculated for cumacean species sampled by a sledge in the 10-40, 45-85, 90-110 and $115-145 \mathrm{~cm}$ near-bottom water layers during the ESSAIS and ECOFER surveys. $\sum \mathrm{Nt}$, total number of specimens (cumulative values from all available water layers and stations, excluding TS09).

\begin{tabular}{|c|c|c|c|c|c|}
\hline Taxa & $\sum \mathrm{Nt}$ & K1 & $\mathrm{K} 2$ & $\mathrm{~K} 3$ & K4 \\
\hline \multicolumn{6}{|l|}{ Family BODOTRIIDAE } \\
\hline Bathycuma brevirostre & 11 & 1.00 & 0.00 & 0.00 & 0.00 \\
\hline Cyclaspis longicaudata & 26 & 0.88 & 0.12 & 0.00 & 0.00 \\
\hline Cyclaspoides sarsi & 8 & 1.00 & 0.00 & 0.00 & 0.00 \\
\hline Iphinoe serrata & 1 & 0.00 & 0.00 & 1.00 & 0.00 \\
\hline \multicolumn{6}{|l|}{ Family DIASTYLIDAE } \\
\hline Diastylis cornuta & 2 & 0.50 & 0.00 & 0.00 & 0.50 \\
\hline Diastylis tumida & 6 & 1.00 & 0.00 & 0.00 & 0.00 \\
\hline Diastyloides biplicatus & 33 & 0.94 & 0.06 & 0.00 & 0.00 \\
\hline Diastyloides serratus & 128 & 0.72 & 0.13 & 0.08 & 0.07 \\
\hline Leptostylis longimana & 6 & 1.00 & 0.00 & 0.00 & 0.00 \\
\hline Leptostylis macrura & 133 & 0.97 & 0.02 & 0.01 & 0.00 \\
\hline Leptostylis sp.A & 3 & 1.00 & 0.00 & 0.00 & 0.00 \\
\hline Leptostylis sp.B & 1 & 1.00 & 0.00 & 0.00 & 0.00 \\
\hline Makrokylindrus (A.) anomalus & 1 & 1.00 & 0.00 & 0.00 & 0.00 \\
\hline Makrokylindrus (A.) josephinae & 123 & 0.93 & 0.04 & 0.03 & 0.00 \\
\hline Makrokylindrus (A.) longicaudatus & 8 & 0.88 & 0.13 & 0.00 & 0.00 \\
\hline Makrokylindrus (A.) longipes & 7 & 1.00 & 0.00 & 0.00 & 0.00 \\
\hline Vemakylindrus hastatus & 39 & 1.00 & 0.00 & 0.00 & 0.00 \\
\hline \multicolumn{6}{|l|}{ Family LAMPROPIDAE } \\
\hline Hemilamprops normani & 28 & 0.93 & 0.04 & 0.00 & 0.04 \\
\hline Mesolamprops denticulatus & 72 & 0.82 & 0.08 & 0.07 & 0.03 \\
\hline pus typicus & 12 & 1.00 & 0.00 & 0.00 & 0.00 \\
\hline Platytyphlops orbicularis & 4 & 1.00 & 0.00 & 0.00 & 0.00 \\
\hline \multicolumn{6}{|l|}{ Family LEUCONIDAE } \\
\hline Eudorella cf. parvula & 26 & 0.96 & 0.00 & 0.04 & 0.00 \\
\hline Ithyleucon sorbei & 18 & 0.94 & 0.06 & 0.00 & 0.00 \\
\hline Leucon $(C$.$) tener$ & 9 & 1.00 & 0.00 & 0.00 & 0.00 \\
\hline Leucon (E.) ensis & 11 & 1.00 & 0.00 & 0.00 & 0.00 \\
\hline Leucon (E.) pusillus & 87 & 0.98 & 0.01 & 0.01 & 0.00 \\
\hline Leucon (L.) affinis & 19 & 0.89 & 0.05 & 0.05 & 0.00 \\
\hline (L.) sp. & 11 & 1.00 & 0.00 & 0.00 & 0.00 \\
\hline Leucon $(M$.$) siphonatus$ & 31 & 0.87 & 0.06 & 0.06 & 0.00 \\
\hline \multicolumn{6}{|l|}{ Family NANNASTACIDAE } \\
\hline Campylaspis glabra & 84 & 0.9 & 0.06 & 0.01 & 0.02 \\
\hline Campylaspis laevigata & 123 & 0.95 & 0.04 & 0.01 & 0.00 \\
\hline Campylaspis nitens & 8 & 1.00 & 0.00 & 0.00 & 0.00 \\
\hline Campylaspis rostrata & 2 & 1.00 & 0.00 & 0.00 & 0.00 \\
\hline Campylaspis squamifera & 368 & 0.91 & 0.07 & 0.01 & 0.00 \\
\hline Campylaspis sulcata & 42 & 0.98 & 0.02 & 0.00 & 0.00 \\
\hline Cumella (C.) divisa & 1 & 1.00 & 0.00 & 0.00 & 0.00 \\
\hline Cumellopsis puritani & 9 & 1.00 & 0.00 & 0.00 & 0.00 \\
\hline s atlanticus & 2 & 1.00 & 0.00 & 0.00 & 0.00 \\
\hline Procampylaspis armata & 85 & 0.95 & 0.02 & 0.01 & 0.01 \\
\hline Procampylaspis omnidion & 4 & 1.00 & 0.00 & 0.00 & 0.00 \\
\hline Schizocuma spinoculatum & 11 & 1.00 & 0.00 & 0.00 & 0.00 \\
\hline Styloptocuma gracillimum & 50 & 0.98 & 0.00 & 0.02 & 0.00 \\
\hline Global & 1653 & 0.92 & 0.05 & 0.02 & 0.01 \\
\hline
\end{tabular}



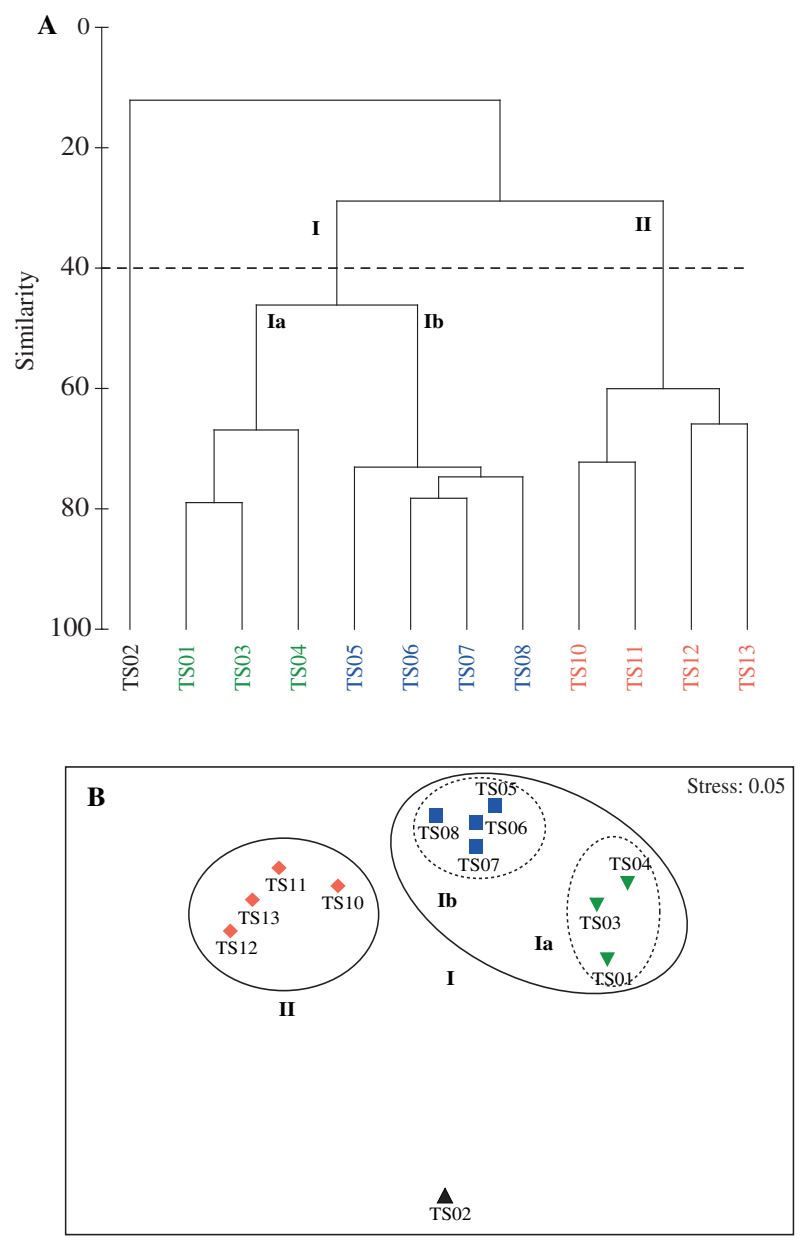

Fig. 3. - Hierarchical cluster analysis (A) and non-metric multidimensional scaling (B) of the 12 slope stations (TS09 excluded; see text) based on square-root transformed abundances and Bray-Curtis similarity measure. are concentrated close to the seafloor during both daytime and night-time (see the case of the night-time samples TS06 and TS13). Furthermore, both families and species showed a drastic abundance decrease between the N1 and N2 lowermost waters layers and species richness showed the same vertical decreasing trend (see Table 4 and S1). Twenty-one species were exclusively sampled in the 10-40 cm water layer and therefore had a K1 index of 1: Bathycuma brevirostre, Cyclaspoides sarsi, Diastylis tumida, Leptostylis longimana, Leptostylis sp.A, Leptostylis sp.B, Makrokylindrus (Adiastylis) anomalus, M. (A.) longipes, Vemakylindrus hastatus, Platysympus typicus, Platytyphlops orbicularis, Leucon (Crymoleucon) tener, Leucon (Epileucon) ensis and Leucon (Leucon) sp., Campylaspis nitens, C. rostrata, Cumella (Cumella) divisa, Cumellopsis puritani, Nannastacus atlanticus, Procampylaspis omnidion and Schizocuma spinoculatum. The other species showed high K1 values (range: 0.50-0.98) in the lowermost level and lower K2-K4 decreasing values (range: $0.33-0$ ) in the upper ones. Only six species were also sampled in the $115-145 \mathrm{~cm}$ water layer, with a generally very low $\mathrm{K} 4$ index, probably attesting their higher swimming abilities than the preceding ones: Diastylis cornuta, Diastyloides serratus; Hemilamprops normani, Mesolamprops denticulatus; Campylaspis glabra and Procampylaspis armata. Iphinoe serrata, a shelf-origin bodotriid, was sporadically sampled in the $80-110 \mathrm{~cm}$ water layer of station TS02 (only one specimen caught).

As presented in Figure 3, the cluster analysis carried out on abundance data reveals the existence of three main groups of stations distributed over depth (station TS02 being outside the groups), and the multidimensional scaling ordination shows similar results to those of the dendrogram, with an excellent stress

Table 5. - Average dissimilarity between the 3 main station groups discriminated by the multivariate analysis of the 12 sampling stations (station TS02 discarded) and contribution of cumacean species to total dissimilarity. A cut-off at a cumulative dissimilarity of $85 \%$ was applied.

\begin{tabular}{|c|c|c|c|c|c|}
\hline $\begin{array}{l}\text { Groups Ia } v s \mathrm{Ib} \\
\text { Av. diss.: } 63.9\end{array}$ & $\%$ & $\begin{array}{l}\text { Groups Ia vs II } \\
\text { Av. diss.: } 86.85\end{array}$ & $\%$ & $\begin{array}{l}\text { Groups Ib vs II } \\
\text { Av. diss.: } 70.09\end{array}$ & $\%$ \\
\hline $\begin{array}{l}\text { Campylaspis squamifera } \\
\text { Campylaspis laevigata } \\
\text { Procampylaspis armata } \\
\text { Campylaspis sulcata } \\
\text { Campylaspis glabra } \\
\text { Mesolamprops denticulatus } \\
\text { Diastyloides biplicatus } \\
\text { Leucon (L.) affinis } \\
\text { Styloptocuma gracillimum } \\
\text { Diastyloides serratus } \\
\text { Leptostylis macrura } \\
\text { Makrokylindrus (A.) josephinae }\end{array}$ & $\begin{array}{l}20.73 \\
12.10 \\
7.46 \\
6.59 \\
6.14 \\
6.10 \\
5.09 \\
5.03 \\
4.93 \\
4.81 \\
3.86 \\
3.60\end{array}$ & $\begin{array}{l}\text { Makrokylindrus (A.) josephinae } \\
\text { Leucon (E.) pusillus } \\
\text { Procampylaspis armata } \\
\text { Hemilamprops normani } \\
\text { Vemakylindrus hastatus } \\
\text { Cyclaspis longicaudata } \\
\text { Diastyloides serratus } \\
\text { Leucon (M.) siphonatus } \\
\text { Campylaspis sulcata } \\
\text { Leptostylis macrura } \\
\text { Eudorella cf. parvula } \\
\text { Campylaspis squamifera } \\
\text { Bathycuma brevirostre } \\
\text { Leucon (C.) tener } \\
\text { Ithyleucon sorbei } \\
\text { Campylaspis laevigata } \\
\text { Leucon (L.) sp. } \\
\text { Mesolamprops denticulatus } \\
\text { Platytyphlops orbicularis } \\
\text { Diastylis tumida } \\
\text { Leucon (E.) ensis } \\
\text { Styloptocuma gracillimum } \\
\text { Campylaspis glabra } \\
\text { Diastyloides biplicatus }\end{array}$ & $\begin{array}{l}9.90 \\
7.95 \\
5.90 \\
5.15 \\
5.00 \\
4.86 \\
4.70 \\
4.32 \\
4.30 \\
3.63 \\
3.03 \\
2.60 \\
2.53 \\
2.53 \\
2.46 \\
2.29 \\
2.28 \\
2.17 \\
2.00 \\
1.94 \\
1.86 \\
1.75 \\
1.66 \\
1.35\end{array}$ & $\begin{array}{l}\text { Campylaspis squamifera } \\
\text { Leucon (E.) pusillus } \\
\text { Makrokylindrus (A.) josephinae } \\
\text { Leptostylis macrura } \\
\text { Mesolamprops denticulatus } \\
\text { Campylaspis laevigata } \\
\text { Hemilamprops normani } \\
\text { Cyclaspis longicaudata } \\
\text { Campylaspis glabra } \\
\text { Vemakylindrus hastatus } \\
\text { Procampylaspis armata } \\
\text { Leucon (L.) affinis } \\
\text { Styloptocuma gracillimum } \\
\text { Diastyloides serratus } \\
\text { Eudorella cf. parvula } \\
\text { Leucon }(\text { M.) siphonatus } \\
\text { Bathycuma brevirostre } \\
\text { Ithyleucon sorbei } \\
\text { Leucon (C.) tener } \\
\text { Diastyloides biplicatus } \\
\text { Leucon (L.) sp. } \\
\text { Platytyphlops orbicularis } \\
\text { Leucon (E.) ensis } \\
\text { Diastylis tumida } \\
\text { Platysympus typicus }\end{array}$ & $\begin{array}{l}8.85 \\
6.81 \\
6.63 \\
5.09 \\
4.98 \\
4.40 \\
4.38 \\
4.15 \\
3.92 \\
3.78 \\
3.52 \\
2.55 \\
2.55 \\
2.52 \\
2.47 \\
2.44 \\
2.20 \\
2.16 \\
2.15 \\
1.98 \\
1.98 \\
1.70 \\
1.63 \\
1.61 \\
1.30\end{array}$ \\
\hline
\end{tabular}


Table 6. - Biotic characteristics of the cumacean assemblages Ia, Ib and II discriminated by the multivariate analysis of abundance data (based on cumulative values from nets N1-N4). Species ranked according to their average contribution to within-group similarity (\%Sim); their respective contribution $(\% \mathrm{D})$ to mean total abundance of each assemblage is also included. A cut-off at a cumulative similarity of $80 \%$ was applied; sd, standard deviation.

\begin{tabular}{|c|c|c|c|c|}
\hline \multicolumn{2}{|l|}{$\begin{array}{r}\text { Assemblages } \\
\text { Haul codes TS } \\
\text { Depth range }(\mathrm{m})\end{array}$} & $\begin{array}{c}\mathrm{Ia} \\
1,3,4 \\
346-485\end{array}$ & $\begin{array}{c}\mathrm{Ib} \\
5,6,7,8 \\
522-714\end{array}$ & $\begin{array}{c}\text { II } \\
10,11,12,13 \\
790-1099\end{array}$ \\
\hline \multirow[t]{3}{*}{ Species richness $(\mathrm{S})$} & range & $5-7$ & $13-17$ & $19-25$ \\
\hline & cumulative & 13 & 22 & 35 \\
\hline & mean \pm sd & $6.3 \pm 1.2$ & $14.8 \pm 2.1$ & $22.0 \pm 2.4$ \\
\hline \multirow{2}{*}{ Abundance (ind./100 $\mathrm{m}^{2}$ ) } & range & $2.8-10.1$ & $26.8-55.8$ & $30.8-49.9$ \\
\hline & mean \pm sd & $6.4 \pm 3.7$ & $41.2 \pm 14.2$ & $42.3 \pm 8.7$ \\
\hline \multirow{2}{*}{ Diversity (H', $\left.\log _{2}\right)$} & range & $1.98-2.37$ & $2.76-3.05$ & $3.53-4.05$ \\
\hline & Mean \pm sd & $2.15 \pm 0.20$ & $2.89 \pm 0.12$ & $3.78 \pm 0.25$ \\
\hline \multirow[t]{2}{*}{ Evenness (J') } & range & $0.75-0.85$ & $0.67-0.83$ & $0.81-0.88$ \\
\hline & mean \pm sd & $0.81 \pm 0.06$ & $0.75 \pm 0.07$ & $0.85 \pm 0.03$ \\
\hline \multirow[t]{11}{*}{$\begin{array}{l}\text { Main species } \\
\text { (\% Sim / \%D) }\end{array}$} & & $\begin{array}{l}\text { Leptostylis macrura } \\
(36.4 / 28.1)\end{array}$ & $\begin{array}{c}\text { Campylaspis squamifera } \\
(24.7 \text { / 34.4) }\end{array}$ & $\begin{array}{c}\text { Makrokylindrus (A.) josephinae } \\
(15.8 / 17.2)\end{array}$ \\
\hline & & $\begin{array}{l}\text { Diastyloides serratus } \\
(14.7 / 8.9)\end{array}$ & $\begin{array}{c}\text { Campylaspis laevigata } \\
(13.6 / 11.3)\end{array}$ & $\begin{array}{c}\text { Leucon pusillus } \\
(10.9 / 12.6)\end{array}$ \\
\hline & & $\begin{array}{c}\text { Campylaspis glabra } \\
(14.1 / 8.3)\end{array}$ & $\begin{array}{l}\text { Leptostylis macrura } \\
\quad(11.2 / 10.6)\end{array}$ & $\begin{array}{c}\text { Diastyloides serratus } \\
(10.8 / 10.2)\end{array}$ \\
\hline & & $\begin{array}{c}\text { Mesolamprops denticulatus } \\
(14.1 / 5.7)\end{array}$ & $\begin{array}{c}\text { Campylaspis glabra } \\
(11.0 / 6.9)\end{array}$ & $\begin{array}{c}\text { Procampylaspis armata } \\
(7.8 / 6.3)\end{array}$ \\
\hline & & $\begin{array}{c}\text { Campylaspis sulcata } \\
(12.7 / 34.9)\end{array}$ & $\begin{array}{c}\text { Mesolamprops denticulatus } \\
(8.2 / 7.4)\end{array}$ & $\begin{array}{c}\text { Hemilamprops normani } \\
(7.5 / 4.8)\end{array}$ \\
\hline & & & $\begin{array}{c}\text { Styloptocuma gracillimum } \\
(7.1 / 4.3)\end{array}$ & $\begin{array}{c}\text { Cyclaspis longicaudata } \\
(7.0 / 4.4)\end{array}$ \\
\hline & & & $\begin{array}{c}\text { Diastyloides serratus } \\
(7.0 / 6.5)\end{array}$ & $\begin{array}{c}\text { Leucon }(M .) \text { siphonatus } \\
(6.5 / 3.4)\end{array}$ \\
\hline & & & & Vemakylindrus hastatus \\
\hline & & & & $\begin{array}{c}\text { Platytyphlops orbicularis } \\
(3.1 / 0.7)\end{array}$ \\
\hline & & & & $\begin{array}{c}\text { Campylaspis squamifera } \\
(2.8 / 2.5)\end{array}$ \\
\hline & & & & $\begin{array}{c}\text { Leucon }(C .) \text { tener } \\
(2.5 / 1.6)\end{array}$ \\
\hline
\end{tabular}

value of 0.05 . As shown in Table 5 , the average dissimilarity between these groups was $\geq 63.9$, with the highest value recorded between groups Ia and II. Two nannastacids mainly contributed to the total dissimilarity between groups Ia and Ib: Campylaspis squamifera $(20.73 \%)$ and C. laevigata (12.10\%). Dissimilarity between other paired groups was due to a higher number of contributing species, with Makrokylindrus (Adiastylis) josephinae $(9.90 \%)$ and Campylaspis squamifera $(8.85 \%)$ as the top species for paired groups Ia-II and Ib-II, respectively.

Group Ia (3 stations between 346 and 485 m depth; average within-group similarity 63.8) is characterized by the numerical dominance of Nannastacidae (57.2\%), the absence of Bodotriidae and Leuconidae and a low total abundance of $6.4 \pm 3.7$ ind./100 $\mathrm{m}^{2}$ (mean \pm SD). Leptostylis macrura, Diastyloides serratus, Campylaspis glabra, Mesolamprops denticulatus and Campylaspis sulcata accounted for $92.0 \%$ of the average within-group similarity (Table 6). Campylaspis sulcata was the most abundant species of this assemblage, with a mean value of $2.2 \pm 2.1$ ind./100 $\mathrm{m}^{2}($ mean $\pm \mathrm{SD})$ and a contribution of $34.9 \%$ to total group abundance, followed by Leptostylis macrura (28.1\%), Styloptocuma gracillimum $(9.4 \%)$, Diastyloides serratus $(8.9 \%)$ and Campylaspis glabra $(8.3 \%)$.

Group Ib (4 stations between 522 and 714 m depth; average within-group similarity 71.3) was also characterized by the dominance of Nannastacidae $(66.1 \%)$ and the absence of Bodotriidae but marked by the appear- ance of Leuconidae and a higher total abundance than the preceding one $\left(41.2 \pm 14.2\right.$ ind./100 $\mathrm{m}^{2}$, mean $\left.\pm \mathrm{SD}\right)$. Campylaspis squamifera, C. laevigata, Leptostylis macrura, Campylaspis glabra, Mesolamprops denticulatus, Styloptocuma gracillimum and Diastyloides serratus accounted for $82.8 \%$ of the average within-group similarity (Table 6). Campylaspis squamifera was the most abundant species of this assemblage, with a mean value of $14.2 \pm 3.9$ ind. $/ 100 \mathrm{~m}^{2}($ mean \pm SD) and a contribution of $34.4 \%$ to total group abundance, followed by C. laevigata (11.3\%), Leptostylis macrura (10.6\%), Mesolamprops denticulatus (7.4\%) and Procampylaspis armata $(7.0 \%)$. Three species, Diastylis cornuta, Leucon (Leucon) affinis and Cumellopsis puritani, were found exclusively in this assemblage.

Group II (4 stations between 790 and 1099 m depth; average within-group similarity 61.3 ) was characterized by the dominance of Diastylidae (40.3\%), the appearance of Bodotriidae and a total abundance of $42.3 \pm 8.7$ ind./100 $\mathrm{m}^{2}$ (mean $\left.\pm \mathrm{SD}\right)$. This group showed the highest mean values of species richness, total abundance and diversity indices (Table 6). Makrokylindrus (Adiastylis) josephinae, Leucon (Epileucon) pusillus, Diastyloides serratus, Procampylaspis armata, Hemilamprops normani, Cyclaspis longicaudata, Leucon (Macrauloleucon) siphonatus, Vemakylindrus hastatus, Platytyphlops orbicularis, Campylaspis squamifera and Leucon (Crymoleucon) tener accounted for $81.0 \%$ of the average within-group similarity (Table 6). Makrokylindrus (A.) josephinae was the 
most abundant species of this assemblage, with a mean value of $7.3 \pm 1.5$ ind./100 $\mathrm{m}^{2}$ (mean $\pm \mathrm{SD}$ ) and a contribution of $17.2 \%$ to total group abundance, followed by Leucon (Epileucon) pusillus (12.6\%), Diastyloides serratus (10.2\%), Procampylaspis armata (6.3\%) and Vemakylindrus hastatus (5.4\%). 18 species were found exclusively in this assemblage, demonstrating an evident renewal of the cumacean fauna at this bathymetric level: Bathycuma brevirostre, Cyclaspis longicaudata, Cyclaspoides sarsi, Leptostylis longimana, Leptostylis sp.B, Makrokylindrus (Adiastylis) anomalus, M. (A.) longicaudatus, M. (A.) longipes, Hemilamprops normani, Platytyphlops orbicularis, Ithyleucon sorbei, Leucon (Crymoleucon) tener, Leucon (Epileucon) ensis, L. (E.) pusillus, Campylaspis rostrata, Cumella (Cumella) divisa, Procampylaspis omnidion and Schizocuma spinoculatum.

\section{DISCUSSION}

Lagardère (1977) carried out a pioneering study on the bathyal cumacean fauna of the southeastern Bay of Biscay (composition and bathymetric distribution of species in the depth range 200-1400 m). According to modern nomenclature (see Băcescu 1992, Corbera and Sorbe 1999), we suggest the following equivalence between species (Lagardère's taxa in brackets): Vemakylindrus hastatus (= Diastylis cf. hastata), Eudorella cf. parvula (= E. truncatula), Cumella (Cumella) divisa (= Cumella sp.), Nannastacus atlanticus (= Nannastacus sp.) and Styloptocuma gracillimum (= Cumella gracillimana). Within the restricted depth range 200-1000 m (comparable to the present study), Lagardère listed 37 species belonging to Nannastacidae, Diastylidae, Leuconidae, Bodotriidae and Lampropidae (decreasing order of species richness), slightly lower than the value obtained herein (43 species). Eudorella hirsuta (Sars, 1869) is probably a misidentification (= Eudorella $\mathrm{cf}$. parvula?), as is Campylaspis horrida Sars, 1869 recorded on the upper slope of Arctic waters according to Jones (1984). Although not recorded during the present survey, $\mathrm{He}$ milamprops roseus (Norman, 1863) and Campylaspis macrophthalma Sars, 1878 were sampled on the outer shelf adjacent to our study area (Sorbe 1984); Vaunthompsonia cristata Bate, 1858, Campylaspis verrucosa Sars, 1866 and Campylaspis vitrea Calman, 1906 were sampled in the Capbreton canyon area (Frutos and Sorbe 2014, 2017); and Hemilamprops cristatus (Sars, 1870) and Leucon (Epileucon) longirostris Sars, 1871 were mentioned from bathyal bottoms of the Bay of Biscay (Jones 1985, Frutos and Sorbe 2014). Therefore, except probable misidentified species, all cumaceans mentioned by Lagardère (1977) were found again in more recent studies on the bathyal benthic cumacean fauna of the SE Bay of Biscay. Furthermore, a new genus and species, Ithyleucon sorbei, were described from the material collected during the present study (Corbera 2012), and two apparently undescribed species of the genus Leptostylis remain to be studied.

Previously reported from the NE Atlantic between Norway and British Islands but also from the Azores and the Gulf of Cadiz, Diastylis tumida (740-924 m) is mentioned for the first time in the Bay of Biscay (present study). Originally described from bathyal bottoms of the Gulf of Lion, Mesolamprops denticulatus Ledoyer, 1983 was more recently discovered in the NE Atlantic Ocean as far as the Faeroe-Shetland Channel at 259-753 $\mathrm{m}$ (Shalla and Bishop 2007) as well as in the southern Bay of Biscay (Frutos and Sorbe 2014, Capbreton Canyon; Sorbe and Elizalde 2014, southern margin of the Cap Ferret Canyon).

Up to now, the structure of deep cumacean assemblages has been poorly investigated. In the southern Bay of Biscay, recent studies on bathyal suprabenthic assemblages (Frutos and Sorbe 2014, 2017, Sorbe and Elizalde 2014) showed that they constitute one of the main components of the near-bottom motile fauna (suprabenthic ecophase), in addition to amphipods, isopods and mysids. However, another part of these assemblages is known to inhabit surficial sediments constituting the endobenthic ecophase of these populations (Fage 1951, Jones 1976, Băcescu and Petrescu 1999). These burrowing individuals are inadequately sampled by suprabenthic sledges, mainly designed to sample the near-bottom motile fauna (epi-/suprabenthic ecophase). Therefore, more realistic estimations of benthic cumacean abundances should ideally combine sampling with sledges and grabs/box cores. To our knowledge, such a methodology has never been implemented in the study of these benthic communities. Table 7 shows some structural data on diverse shelf and slope cumacean assemblages, sampled with either suprabenthic sledges or grabs. The abundance data (maximum recorded values for each study) given by suprabenthic sampling are in the same order of magnitude for shelf (range: $1.2-6.5$ ind $\mathrm{m}^{-2}$ ) and slope assemblages (range: 0.3-5.4 ind $\mathrm{m}^{-2}$ ), and the corresponding mean values are statistically equal $\left(\mathrm{t}_{\mathrm{obs}}=1.256 ;\right.$ d.f. $\left.=10 ; \mathrm{p}>0.05\right)$. In shelf assemblages, these suprabenthic abundances are generally much lower than values obtained with grab sampling (range: 153-24000 ind. $\mathrm{m}^{-2}$ ) and far from the worldwide maximum value mentioned by Hawkinson (1992) in the case of the coastal Diastylidae Diastylopsis dawsoni Smith, 1880 from Agate Bay, California (up to 119881 ind. m ${ }^{-2}$; Ekman grab).

According to the structural data presented in Table 7, species richness (maximum values) is generally higher in slope assemblages (range: 7-25 species per station) than in shelf ones (range: $4-15$ species per station). Such a trend is also verified when considering the St mean values for slope $(29.2 \pm 13.0$ species per station; mean \pm SD) and shelf ( $10.9 \pm 6.6$ species per station) assemblages of the studied areas $\left(\mathrm{t}_{\mathrm{obs}}=3.733\right.$; d.f. $=15$; $\mathrm{p}<0.001)$. Furthermore, within the slope assemblages studied herein, the cumulative species richness increases with depth: 13, 22 and 35 species for assemblages Ia, Ib and II, respectively. Although without statistical significance, the diversity values, $\mathrm{H}^{\prime}$, are also higher in slope assemblages (range: $3.41-4.05$ ) than in the shelf ones (range: 1.24-3.21), corroborating previous observations on bathyal cumacean assemblages (Jones and Sanders 1972, Reyss 1973) as well as on all bathyal suprabenthic assemblages (Frutos and Sorbe 2014, 2017, Sorbe and Elizalde 2014). 
Table 7. - Comparison of structural data reported for shelf and slope cumacean assemblages from various geographical areas. Smax/Dmax, maximum value of species richness/abundance recorded at a sampling station in the study area. St, total species richness recorded in the whole study area. H', maximum value of the Shannon-Wiener diversity index recorded at a sampling station of the study area. -, no data. ${ }^{\mathrm{a}}$ unpublished data; ${ }^{\mathrm{b}}$ calculated from rough data mentioned in reference; ${ }^{\mathrm{c}, \mathrm{d}}$ daytime, night-time samplings; ${ }^{\mathrm{e}}$ recalculted from original data in ind. $/ 100 \mathrm{~m}^{3}$ (conversion factor: $0.51510^{-2}$ ).

\begin{tabular}{|c|c|c|c|c|c|c|c|}
\hline Bathymetrical and geographical areas & Sampling gear & $\begin{array}{l}\text { Depth range } \\
(\mathrm{m})\end{array}$ & Smax & St & $\begin{array}{c}\text { Dmax } \\
\text { (ind. } \mathrm{m}^{-2} \text { ) }\end{array}$ & $\begin{array}{c}\mathrm{H}^{\prime} \\
\left(\log _{2}\right)\end{array}$ & References \\
\hline \multicolumn{8}{|l|}{ Shelf areas } \\
\hline Hendaye beach (NE Atlantic) & sledge & $0-0.2$ & - & 5 & - & - & San Vicente and Sorbe 2001 \\
\hline Creixell beach (NW Mediterranean) & sledge & $0.5-3.5$ & - & 6 & - & - & San Vicente and Sorbe 1999 \\
\hline Galician ria (NE Atlantic) & grab & $0-28.2$ & 4 & 4 & 153.6 & - & Cacabelos et al. 2010 \\
\hline Catalan Sea (NW Mediterranean) & grab & 15 & 10 & 1 & 333.0 & - & Corbera et al. 2013 \\
\hline Persian Gulf & grab & $15-30$ & $4^{\mathrm{a}}$ & 8 & $260.0^{\mathrm{a}}$ & $1.24^{\mathrm{a}}$ & Martin et al. 2010 \\
\hline Levantine Sea (E Mediterranean) & grab & $1.9-63$ & 8 & 18 & 24000.0 & 2.04 & Corbera and Galil 2016 \\
\hline Catalan Sea (NW Mediterranean) & grab & $5-70$ & 8 & 22 & 612.0 & $2.58^{\mathrm{b}}$ & Corbera and Cardell 1995 \\
\hline Aquitanian shelf (NE Atlantic) & sledge & $31^{\mathrm{c}}$ & 5 & 7 & $2.4^{\mathrm{e}}$ & 1.69 & Sorbe 1984 \\
\hline$\ll$ & $\ll$ & $91^{\mathrm{c}}$ & 9 & 14 & $3.2^{\mathrm{e}}$ & 2.46 & « \\
\hline « & « & $91^{\mathrm{d}}$ & 10 & 14 & $6.5^{\mathrm{e}}$ & 2.49 & « \\
\hline « & $\ll$ & $126^{\mathrm{c}}$ & 12 & 15 & $1.2^{\mathrm{e}}$ & 3.21 & « \\
\hline$\ll$ & « & $179^{c}$ & 15 & 17 & $4.3^{\mathrm{e}}$ & 2.93 & « \\
\hline \multicolumn{8}{|l|}{ Slope areas } \\
\hline Cap Ferret slope (NE Atlantic) & sledge & $386-420$ & 7 & 9 & 0.3 & - & Sorbe and Elizalde 2014 \\
\hline Capbreton Canyon (NE Atlantic) & sledge & $151-797$ & 12 & 25 & 3.0 & - & Frutos and Sorbe 2017 \\
\hline Kostarrenkala (NE Atlantic) & sledge & $175-1000$ & 24 & 38 & 5.4 & - & Frutos and Sorbe 2014 \\
\hline Cap Ferret slope (NE Atlantic) & sledge & 346-1099 & 25 & 42 & 0.6 & 4.05 & this study \\
\hline Catalan Sea (NW Mediterranean) & sledge & $389-1859$ & 19 & 32 & 2.5 & $3.41^{\mathrm{b}}$ & Cartes and Sorbe 1997 \\
\hline Cap Ferret Canyon (NE Atlantic) & sledge & $2410-2425$ & - & - & 1.7 & - & Sorbe 1999 \\
\hline Cap Ferret Canyon (NE Atlantic) & sledge & $3058-3070$ & - & - & 0.3 & - & $\ll$ \\
\hline
\end{tabular}

According to our multivariate analysis (see Fig. 3), two main faunal changes occur in the cumacean fauna collected on the upper bathyal of the southern Bay of Biscay. The first one is observed between 485 and $522 \mathrm{~m}$, and the second one (more clearly marked) between 714 and $790 \mathrm{~m}$. In the upper part of the study area, the substratum consists of muddy fine sands ( $c a$. $400 \mathrm{~m}$ depth, median grain size $[\mathrm{Mz}]=73.9 \mu \mathrm{m}$; particles $<63 \mu \mathrm{m}$ [pelites] $=38.2 \%$; organic carbon content $\left[\mathrm{C}_{\text {org }}\right]=0.37 \%$ of sediment dry weight; see Etcheber et al 1999 and Elizalde 1994). The edge of the adjacent shelf is characterized by comparable bottoms (ca. 179 m depth: $\mathrm{Mz}=100.0 \mu \mathrm{m}$; pelites $=20.0 \%$; see Sorbe 1984). This similarity in the texture of surficial sediments favours the extension of some shelf species down to the upper bathyal (for instance the Bodotriidae Iphinoe serrata and the Nannastacidae Nannastacus atlanticus). Separating the cumacean assemblages Ia and $\mathrm{Ib}$, the upper ecotone at about $500 \mathrm{~m}$ depth is related to the apparition of muddy sediments (characterized by a pelitic fraction $\geq 50 \%$; see Elizalde 1994) that are more fluid than the shallower sandy bottoms. This structural change on the sedimentary coverage has a probable effect on the functioning of the suprabenthic sledge. Primarily designed to slide on compact sandy bottoms thanks to its lateral skates (see Dauvin et al. 1995), this device probably skims the uppermost sediment layer on fluid muddy bottoms and thus samples surficial infaunal components in addition to the suprabenthic ones. This phenomenon is attested by a significant increase in cumacean mean abundances below 500 m depths: $42.7 \pm 10.6$ ind./100 $\mathrm{m}^{2}$ (mean \pm SD) versus $5.6 \pm 3.4$ ind.$/ 100 \mathrm{~m}^{2}$ at shallower depths $\left(\mathrm{t}_{\mathrm{obs}}=48.4\right.$; d.f. $=11 ; \mathrm{p}<0.001)$. It should be noted that no Leuconidae were observed within the assemblage Ia (346-485 m), although Leucon (Macrauloleucon) siphonatus curiously showed a disjoint bathymetric distribution, being present on both muddy sand bottoms of the outer shelf (91-179 m; Sorbe 1984) and deeper muddy slope bottoms (608-1099 m; this study).

These new observations corroborate the eurybathic (100-4380 m; Fage 1951) and eurytopic distribution of this strange cumacean with an unusually long branchial siphon (longer than the carapace in some adults; Fage 1951). This morphological peculiarity is probably an adaptive character allowing this species to colonize very diverse benthic habitats. The lower ecotone is imprecisely located between 714 and $791 \mathrm{~m}$ depth (due to absence of detailed data for the cumacean fauna of haul TS09). It constitutes the upper limit of the cumacean assemblage II installed on muddy bottoms characterized by a higher organic carbon content of surficial sediments $\left(\mathrm{C}_{\mathrm{org}}\right.$ from $0.84 \%$ at $720 \mathrm{~m}$ to $1.36 \%$ at $995 \mathrm{~m}$ ) and a pelitic fraction $\geq 84 \%$ (see Etcheber et al. 1999). At ca. $1099 \mathrm{~m}$ (the lower limit of the present study area), the surficial sediments show the following features: $\mathrm{Mz}=12.3 \mu \mathrm{m}$; pelites $=92.6 \%$, mainly constituted by particles $<15 \mu \mathrm{m}$ (clay $=58.0 \%) ; \mathrm{C}_{\text {org }}=1.30 \%$ (see Elizalde 1994). This assemblage probably extends on deeper bathyal bottoms because it is composed of many bathyal/abyssal species (not found at shallower depths in the present study), such as Bathycuma brevirostre (350-1700 m), Cyclaspis longicaudata (189$3350 \mathrm{~m}$ ) and Cyclaspoides sarsi (698-1099 m); Makrokylindrus (Adiastylis) anomalus $(950-1550 \mathrm{~m}), M$. (A.) longicaudatus (650-1287 m) and M. (A.) longipes (15-1227 m); Hemilamprops normani $(220-3000 \mathrm{~m})$ and Platytyphlops orbicularis (423-1739 m); Leucon (Crymoleucon) tener (790-1445 m), Leucon (Epileucon) ensis (790-2006 $\mathrm{m}$ ) and L. (E.) pusillus (610-1780 $\mathrm{m})$; and Campylaspis rostrata (220-2338 m), Cumella (Cumella) divisa (610-2864 m), Procampylaspis omnidion (860-4749 m) and Schizocuma spinoculatum (1097-2900 m) (distributional data according to Sars 1899, Hansen 1920, Fage 1951, Bishop 1981b, Jones 1984, Gerken 2018). 
In addition to these surficial sedimentary characters that certainly play a major role in the bathymetric distribution and local abundance of cumaceans, the bathyal bottoms investigated during the present study are impacted by the benthic impingement of two superimposed water bodies: the Eastern North Atlantic Water between 200 and $600 \mathrm{~m}$ and Mediterranean Overflow Water between 700 and $1300 \mathrm{~m}$ (Durieu de Madron et al. 1999). Inferred from CTD profiles carried out in the water column inside the Cap Ferret Canyon (Durieu de Madron et al. 1999) as well as on its southern margin (unpublished data collected during various oceanographic cruises in all seasons except winter), hydrographic conditions in the near-bottom bathyal environment can be supposedly described as follows in order to assess their possible role in the distribution and structuration of the cumacean assemblages detected across the upper slope. Assemblage Ia (346-485 m) is bathed by the Eastern North Atlantic Water, corresponding to near-bottom waters with a salinity range of 35.52 to 5.74 , a temperature range of 10.5 to $11.3^{\circ} \mathrm{C}$ and an oxygen concentration range of 4.16 to $4.48 \mathrm{ml}$ $\mathrm{L}^{-1}$. Assemblage $\mathrm{Ib}(522-754 \mathrm{~m})$ is also bathed by the same water body, corresponding to near-bottom waters with a salinity range of 35.54 to 35.85 , a temperature range of 10.1 to $10.7^{\circ} \mathrm{C}$ and an oxygen concentration range of 3.76 to $4.16 \mathrm{ml} \mathrm{L}^{-1}$. Assemblage II (790$1099 \mathrm{~m}$ ) is under the influence of the Mediterranean Overflow Water, corresponding to near-bottom waters with a salinity range of 35.69 to 35.77 , a temperature range of 9.1 to $10.1^{\circ} \mathrm{C}$ and an oxygen concentration range of 3.65 to $3.69 \mathrm{ml} \mathrm{L}^{-1}$. Whatever the bathymetric level in the upper bathyal, near-bottom temperatures show weak fluctuations during the annual cycle $\left(\leq 1^{\circ} \mathrm{C}\right.$; inferred from seasonal CTD profiles). This environmental variable is probably not the triggering factor of reproductive mechanisms in these cumaceans, as is the case with some peracarid and decapod populations from adjacent shelf waters (Sorbe 1984, San Vicente and Sorbe 2013). Furthermore, even in the case of the Mediterranean waters (minimum $\mathrm{O}_{2}$ concentration: $3.60 \mathrm{ml} \mathrm{L}^{-1}$ ), the near-bottom waters are far from dysoxia as defined by Levin (2003) for oceanic waters $\left(\leq 1.0 \mathrm{ml} \mathrm{L}^{-1}\right)$, with $\mathrm{O}_{2}$ saturation values $\geq 57.24 \%$. As pointed out by Diaz and Rosenberg (1995), the actual $\mathrm{O}_{2}$ values are probably lower at the water-sediment interface, but the alongslope bottom currents known to periodically occur in the study area (Sorbe and Weber 1995, Durieu de Madron et al. 1999) promote the renewal of their near-bottom waters and probably prevent the occurrence of dysoxic bottom events. Finally, it should be noted that the actual impact of these hydrographic variables on the biology and behaviour of benthic peracaridans is far from being well understood.

Although little is known about the diet of cumaceans (see Jones 1976, Băcescu and Petrescu 1999, Błażewicz-Paszkowycz and Ligowski 2002), the distribution of some species could be related to food availability in the bottom environment. The nannastacid species belonging to genera Campylaspis and Procampylaspis are thought to be predators on foraminifers and small crustaceans, based on the structure of their mouthparts modified as piercing organs and on the analysis of their gut contents (Jones 1976, Błażewicz-Paszkowycz and Ligowski 2002). In the southern Bay of Biscay, the noteworthy numerical dominance of Campylaspis species between 425 and $714 \mathrm{~m}$ (see Table S1) is concomitant with the abundance of some benthic agglutinated foraminifers at the same bathymetric level, as reported by Elizalde et al. (1999). Elizalde et al. (1999) showed that the agglutinated foraminifer Pseudoclavulina mexicana (Cushman, 1922) is preyed upon by the isopod Munnopsurus atlanticus (Bonnier, 1896), crushed by the robust mandibles of this species. Such a trophic link with benthic agglutinated foraminifers remains to be demonstrated in the case of the nannastacid species from the southern Bay of Biscay. The other cumaceans taxa herein mentioned are generally classified as detritivores (see Błażewicz-Paszkowycz and Ligowski 2002 for Antarctic species). The development of their bathyal populations is dependent on periodical inputs of nutritive particles originating from the euphotic zone (overlying pelagial waters and/or adjacent shelf areas).

The present results on the bathyal cumacean fauna can be compared with previous published analyses carried out on the other peracarids from the same collection data (346-1099 m), such as the Mysidacea (Elizalde et al. 1991; obsolete denomination, grouping current orders Lophogastrida and Mysida) and the Amphipoda (Dauvin and Sorbe 1995). In each of these bathyal taxocœnoses, three assemblages have been detected related to depth (assemblages A, B and C for convenience of presentation). The ecotone separating the uppermost assemblages A and B is located around $500 \mathrm{~m}$ depth for the three taxocœnoses. As demonstrated herein for cumaceans, these faunal changes are mainly related to a modification of the sedimentary cover (correlated to peculiar hydrodynamic conditions at the shelf break/upper slope), from muddy sands in the upper levels to muddy bottoms below $500 \mathrm{~m}$ depth. The upper assemblages A are partly composed of shelf species extending their bathymetric distribution to the muddy sand bottoms of the upper slope (for instance, the amphipods Amphilochoides boecki G.O. Sars, 1892, Rhachotropis integricauda Carausu, 1948, Iphimedia obesa Rathke, 1843, Hippomedon denticulatus (Spence Bate, 1857), Westwoodilla caecula (Spence Bate, 1857), Apherusa bispinosa (Spence Bate, 1857) and A. ovalipes Norman and Scott, 1906; and the mysids Leptomysis gracilis (G.O. Sars, 1864) and Mysideis parva Zimmer, 1915). The bathymetric location of the lower ecotone separating assemblages B and C is variable according to taxocœnoses, probably reflecting different sensibility of taxa /species to near-bottom environmental changes. For cumaceans and amphipods, this ecotone is located between 714 and $754 \mathrm{~m}$ (Dauvin and Sorbe 1995; this study), suggesting that some amphipods are also affected by the increase in organic carbon in muddy surficial sediments, as mentioned above for cumaceans. For mysids, this lower ecotone is significantly located deeper on the slope (between 924 and $1024 \mathrm{~m}$ ), probably related to the oxygen minimum 
zone $\left(\mathrm{OMZ} ; \mathrm{O}_{2}\right.$ concentration minimum $=3.6 \mathrm{ml} \mathrm{L}^{-1}$ ) detected between 908 and 942 m on CTD profiles carried out in the study area (see Durieu de Madron et al. 1999). According to available distributional data (Nouvel and Lagardère 1976, Lagardère and Nouvel 1980, Elizalde et al. 1991), this OMZ actually constitutes the lower bathymetric limit of some upper bathyal mysids such as Amblyops spiniferus Nouvel and Lagardère, 1976, Pseudomma kruppi W. Tattersall, 1909 and Mysidella biscayensis Lagardère and Nouvel, 1980, as well as the upper limit of the deeper bathyal mysid Dactylamblyops thaumatops W. Tattersall, 1907 (the numerically dominant species of the deep mysid assemblage). Surprisingly, this OMZ has apparently no impact on the depth distribution of cumacean assemblages but a slight effect on amphipod assemblages, as suggested by the correspondence analysis of data (see Dauvin and Sorbe 1995). Vaquer-Sunyer and Duarte (2008) showed that hypoxia thresholds vary greatly across marine benthic organisms, crustaceans being the most sensitive, but without detailed information on peracarids. Due to their known higher swimming activity (see Mauchline 1980, Elizalde et al. 1991), mysids probably have higher oxygen requirements than most amphipods and cumaceans.

\section{CONCLUSIONS}

In the upper bathyal of the SE Bay of Biscay, cumaceans constitute an important fraction of the suprabenthic fauna, although they are characterized by a lower diversity and abundance than amphipods (a major group in many near-bottom deep assemblages, as pointed out by Frutos et al. 2017). Within the study area, a minimum of 42 cumacean species were censed (some of them putatively new to science), belonging to 5 families (mainly Nannastacidae and Diastylidae). As for other major taxocœnoses, three across-slope cumacean assemblages were detected, mainly structured by depth and surficial sediments (granulometric composition and organic content). This study is a contribution to a better knowledge of the small near-bottom peracarid fauna of the NE Atlantic slope (a major food resource for demersal fishes; see Sorbe 1981), a deep marine area increasingly impacted by many anthropogenic activities (Levin and Dayton 2009).

\section{ACKNOWLEDGEMENTS}

The authors thank the crews of the RV Côte d'Aquitaine (CNRS) and RV Le Noroit (IFREMER), as well as all participants of the oceanographic surveys for their assistance at sea. They wish to express their gratitude to M. Elizalde, J.C. Dauvin and J.C. Lorgeré for their valuable help during sorting of organisms, to J.M. Jouanneau and O. Weber for the communication of hydrological and sedimentological data and also to J.A. Cuesta, I. Frutos and an anonymous reviewer for his comments that improved our manuscript. This study was partially supported by CNRS-CIRMAT (oceanographic cruises and loan of the Roscoff suprabenthic sledge).
Just after finishing the redaction of the last draft of this manuscript, Jean Claude Sorbe, one of the authors, passed away. Thus, this is one of his last contributions to science, to which he dedicated an important part of his life. Those of us who work with him know his tireless passion for work and his rigour, and we acknowledge all his teachings, but above all we appreciate his friendship. He was a great man, and we will not forget him.

\section{REFERENCES}

Băcescu M. 1992. Cumacea II: (Fam. Nannastacidae, Diastylidae, Pseudocumatidae, Gynodiastylidae et Ceratocumatidae). Crustaceorum Catalogus, Pars 8. SPB Academic Publishing, The Hague, pp. 175-468.

Băcescu M., Petrescu I. 1999. Ordre des Cumacés (Cumacea Krøyer, 1846). In: Forest, J. et al. Traité de zoologie: anatomie, systématique, biologie: VII. Crustacés: III A. Péracarides. Mém. Inst. Océanogr. (Monaco) 19: 391-428.

Bishop J.D.D. 1981a. Two new leuconids (Peracarida, Cumacea) of widespread occurrence in the deep Atlantic. Crustaceana 40: 144-159.

https://doi.org/10.1163/156854081X00552

Bishop J.D.D. 1981b. A revised definition of the genus Epileucon Jones (Crustacea, Cumacea), with descriptions of species from the deep Atlantic. Philos. Trans. R. Soc. Lond. B 291: 353-409. https://doi.org/10.1098/rstb.1981.0003

Błażewicz-Paszkowycz M., Ligowski R. 2002. Diatoms as food source indicator for some Antarctic Cumacea and Tanaidacea (Crustacea). Antarct. Sci. 14: 11-15. https://doi.org/10.1017/S0954102002000524

Bonnier J.J. 1896. Édriophthalmes. Résultats Scientifiques de la Campagne du «Caudan» dans le Golfe de Gascogne, aoûtseptembre 1895. Ann. Univ. Lyon 26: 528-562.

Brunel P. 1972. The Gaspe cod ecosystem in the Gulf of St. Lawrence. III. The daily and seasonal vertical migrations of cod (Gadus morhua) in 1960-62. Nat. Can. 99: 287-357.

Cacabelos E., Lourido A., Troncoso J.S. 2010 Composition and distribution of subtidal and intertidal crustacean assemblages in soft-bottoms of the Ría de Vigo (NW Spain). Sci. Mar. 74: 455-464. https://doi.org/10.3989/scimar.2010.74n3455

Cartes J.E., Sorbe J.-C. 1997. Bathyal cumaceans of the Catalan Sea (North-western Mediterranean): faunistic composition, diversity and near-bottom distribution along the slope (between 389 and $1859 \mathrm{~m})$. J. Nat. Hist. 31: 1041-1054. https://doi.org/10.1080/00222939700770541

Clark K.R., Gorley R.N. 2001. Primer v5: User Manual/Tutorial. Plymouth Marine Laboratory.

Corbera J. 2012. Rare and new cumaceans (Crustacea, Peracarida) from the southern margin of the Cap Ferret Canyon (Bay of Biscay). ZooKeys 235: 73-85. https://doi.org/10.3897/zookeys.235.4027

Corbera J., Cardell M.J. 1995. Cumaceans as indicators of eutrophication on soft bottoms. Sci. Mar. 59: 63-69

Corbera J., Galil B.S. 2016. Cumacean assemblages on the Levantine shelf (Mediterranean Sea) - spatiotemporal trends between 2005 and 2012. Mar. Biol. Res. 12: 663-672. https://doi.org/10.1080/17451000.2016.1169297

Corbera J., Sorbe J.C. 1999. The problematic cumacean Schizotrema atlanticum from the eastern Atlantic: redescription and ecological notes. J. Crustac. Biol. 19: 123-130. https://doi.org/10.1163/193724099X00321

Corbera J., Martin D., Pinedo S., et al. 2013. Cumacean density, biomass and productivity in the sandy bottoms of the Bay of Blanes (western Mediterranean). Cah. Biol. Mar. 54: 63-70.

Dagnelie P. 1975. Théorie et méthodes statistiques. Volume II: les méthodes de l'inférence statistique. Les Presses Agronomiques de Gembloux, 463 pp.

Dauvin J.-C., Sorbe J.-C. 1995. Suprabenthic amphipods from the southern margin of the Cap-Ferret canyon (Bay of Biscay, Northeastern Atlantic Ocean): abundance and bathymetric distribution. Pol. Arch. Hydrobiol. 42: 441-460.

Dauvin J.-C., Sorbe J.-C, Lorgeré J.-C 1995. Benthic Boundary Layer macrofauna from the upper continental slope and the Cap Ferret canyon (Bay of Biscay). Oceanol. Acta 18: 113-122. 
Diaz R.J., Rosenberg R. 1995. Marine benthic hypoxia: a review of its ecological effects and the behavioural responses of benthic macrofauna. Oceanogr. Mar. Biol. Annu. Rev. 33: 245-303.

Dixon A. 1944. Notes on certain aspects of the biology of Cumopsis goodsiri (Van Beneden) and some other cumaceans in relation to their environment. J. Mar. Biol. Ass. U.K. 26: 61-71. https://doi.org/10.1017/S0025315400014454

Durrieu de Madron X., Castaing P., Nyffeler F., et al.1999. Slope transport of suspended particulate matter on the Aquitanian margin of the Bay of Biscay. Deep-Sea Res. II 46: 2003-2027. https://doi.org/10.1016/S0967-0645(99)00053-3

Elizalde M. 1994. Les communautés suprabenthiques bathyales de la marge sud du canyon du Cap-Ferret (Golfe de Gascogne). $\mathrm{PhD}$ thesis, Université de Bordeaux 1,212 pp.

Elizalde M., Dauvin J.-C, Sorbe J.-C. 1991. Les mysidacés suprabenthiques de la marge sud du canyon du Cap-Ferret (Golfe de Gascogne): répartition bathymétrique et activité natatoire. Ann. Inst. Océanogr. Paris 67: 129-144.

Elizalde M., Sorbe J.-C, Dauvin J.-C 1993a. Las comunidades suprabentónicas batiales del golfo de Vizcaya (margen sur del cañón de Cap-Ferret): composición faunística y estructura. Publ. Espec. Inst. Esp. Oceanogr. 11: 247-258.

Elizalde M., Weber O., Sorbe J.-C. 1993b. Influence des caractères sédimentologiques sur la distribution des crustacés benthiques de la pente atlantique (Golfe de Gascogne; marge sud du canyon du Cap-Ferret). Actes du IIIe Colloque international «Océanographie du Golfe de Gascogne», pp. 269-273.

Elizalde M., Weber O., Pascual A., et al. 1999. Benthic response of Munnopsurus atlanticus (Crustacea, Isopoda) to the carbon content of the near-bottom sedimentary environment on the southern margin of the Cap-Ferret Canyon (Bay of Biscay, northeastern Atlantic Ocean). Deep-Sea Res. II 46: 2331-2344. https://doi.org/10.1016/S0967-0645(99)00065-X

Etcheber H., Relexans J.-C., Beliard M., et al. 1999. Distribution and quality of sedimentary organic matter on the Aquitanian margin (Bay of Biscay). Deep-Sea Res. II 46: 2249-2288. https://doi.org/10.1016/S0967-0645(99)00062-4

Fage L. 1929. Cumacés et Leptostracés provenant des campagnes du Prince Albert 1er de Monaco. Résult. Camp. Sci. Albert 1er Prince Monaco 77: 3-51.

Fage L. 1951. Cumacés. Faune de France. P. Lechevalier.

Forsman B. 1938. Untersuchungen über die Cumaceen des Skagerraks. Zool. Bid. Uppsala 18: 1-161.

Foxon G. 1936. Notes on the natural history of certain sand-dwelling Cumacea. Ann. Mag. Nat. Hist. 17: 377-393. https://doi.org/10.1080/00222933608655133

Frutos I., Sorbe J.C. 2014. Bathyal suprabenthic assemblages from the southern margin of the Capbreton Canyon ("Kostarrenkala" area), SE Bay of Biscay. Deep-Sea Res. II 104: 291-309. https://doi.org/10.1016/j.dsr2.2013.09.010

Frutos I., Sorbe J.C. 2017. Suprabenthic assemblages from the Capbreton area (SE Bay of Biscay). Faunal recovery after a canyon turbidity disturbance. Deep-Sea Res. Part I 130: 36-46. https://doi.org/10.1016/j.dsr.2017.10.007

Frutos I., Brandt A., Sorbe J.C. 2017. Deep-sea suprabenthic communities: the forgotten biodiversity. In: Rossi S., Bramanti L., et al. (eds), Marine Animal Forests: The ecology of benthic biodiversity hotspots. Springer, Cham. pp. 475-503 https://doi.org/10.1007/978-3-319-21012-4_21

Gage J., Tyler P. 1992. Deep-sea biology: a natural history of organisms at the deep-sea floor. Cambridge University Press. https://doi.org/10.1017/CBO9781139163637

Gerken S. 2018. The Lampropidae (Crustacea: Cumacea) of the World. Zootaxa 4428: 1-192. https://doi.org/10.11646/zootaxa.3630.1.1

Hansen H.J. 1920. Crustacea Malacostraca IV. Danish Ingolf Exp., Copenhagen, vol. 3, $86 \mathrm{pp}$

Hawkinson C.A. 1992. Feeding ecology of gray whales in Agate Bay, California, Summers 1990 and 1991. Master's thesis, San José State University, 45 pp.

Jones N.S. 1969. The systematics and distribution of Cumacea from deeps exceeding 200 meters. Galathea Rep. 10: 99-180.

Jones N.S. 1974. Campylaspis species (Crustacea: Cumacea) from the deep Atlantic. Bull. Br. Mus. (Nat. Hist.) Zool. 27: 247-300. https://doi.org/10.5962/bhl.part.22978

Jones, N.S. 1976. British cumaceans. Synopses of the British Fauna, 7. Academic Press.

Jones N.S. 1984. The family Nannastacidae (Crustacea: Cumacea) from the deep Atlantic. Bull. Br. Mus. (Nat. Hist.) Zool. 46: 207-289. https://doi.org/10.5962/bhl.part.15965

Jones N.S. 1985. Distribution of the Cumacea. In: Laubier L., Monniot C. (eds), Peuplements profonds du golfe de Gascogne: campagnes BIOGAS. IFREMER, Brest, pp 429-433.

Jones N.S., Sanders H.L. 1972. Distribution of Cumacea in the deep Atlantic. Deep-Sea Res. 19: 737-745. https://doi.org/10.1016/0011-7471(72)90066-6

Lagardère J.-P. 1977. Recherches sur la distribution verticale et sur l'alimentation des crustacés décapodes benthiques de la pente continentale du golfe de Gascogne. Analyse des groupements carcinologiques. Bull. Cent. Etud. Rech. Sci. Biarritz 2: 367-440.

Lagardère J.-P., Nouvel H. 1980. Les mysidacés du talus continental du golfe de Gascogne. II. Familles des Lophogastridae, Eucopiidae et Mysidae (Tribu des Erythropini exceptée). Bull. Mus. Natn. Hist. Nat, Paris, 4e série, 2, section A, 2: 375-412.

Levin L.A. 2003. Oxygen minimum zone benthos: adaptation and community response to hypoxia. Oceanogr. Mar. Biol. Annu. Rev. 41: 1-45.

Levin L.A., Dayton P.K. 2009. Ecological theory and continental margins: where shallow meets deep. Trends Ecol. Evol. 24: 606-617.

https://doi.org/10.1016/j.tree.2009.04.012

Martin D., Fernandez-Arcaya U., Tirado P., et al. 2010. Relationships between shallow-water cumacean assemblages and sediment characteristics facing the Iranian coast of the Persian Gulf. J. Mar. Biol. Ass. U.K. 90: 125-134. https://doi.org/10.1017/S0025315409990695

Mauchline J. 1980. The biology of mysids and euphausiids. Adv. Mar. Biol. 18, $681 \mathrm{pp}$

Mees J., Jones M.B. 1997. The hyperbenthos. Oceanogr. Mar. Biol. Annu. Rev. 35: 221-255.

Nouvel H., Lagardère J.-P. 1976. Les mysidacés du talus continental du golfe de Gascogne. I. Tribu des Erythropini (genre Erythrops excepté). Bull. Mus. natn. Hist. nat, Paris, 3e série, n414, Zoologie 291: 1243-1324.

Rex M., Etter R. 2010. Deep-sea biodiversity: pattern and scale. Harvard University Press, Cambridge and London.

Rex M., Etter R., Stuart C. 1997. Large-scale patterns of species diversity in the deep-sea benthos. In: Ormond R.F.G., Gage J.D., Angel M.V. (eds) Marine biodiversity: patterns and processes, Cambridge University Press, pp 94-121. https://doi.org/10.1017/CBO9780511752360.006

Reyss D. 1973. Distribution of Cumacea in the deep Mediterranean. Deep-Sea Res. 20: 1119-1123. https://doi.org/10.1016/0011-7471(73)90025-9

Reyss D. 1974a. Contribution à l'étude des cumacés de profondeur de 1'Atlantique nord: le genre Makrokylindrus Stebbing. Crustaceana 26: 5-28. https://doi.org/10.1163/156854074X00028

Reyss D. 1974b. Contribution à l'étude des cumacés de profondeur en Atlantique: le genre Diastyloides Sars, 1900. Crustaceana 27: $285-293$. https://doi.org/10.1163/156854074X00811

Reyss D. 1978. Cumacés de profondeur de l'Atlantique nord. Famille des Lampropidae. Crustaceana 35: 1-21. https://doi.org/10.1163/156854078X00150

Sainte-Marie B., Brunel P. 1985. Suprabenthic gradients of swimming activity by cold-water gammaridean amphipod Crustacea over a muddy shelf in the Gulf of Saint Lawrence. Mar. Ecol. Prog. Ser. 23: 57-69. https://doi.org/10.3354/meps023057

San Vicente C., Sorbe J.-C. 1999. Spatio-temporal structure of the suprabenthic community from Creixell beach (western Mediterranean). Acta Oecol. 20: 377-389. https://doi.org/10.1016/S1146-609X(99)00129-0

San Vicente C., Sorbe J.-C. 2001. Temporal changes in the structure of the suprabenthic community from Hendaya beach (southern Bay of Biscay): A comparison with a northwestern Mediterranean beach community. Bol. Inst. Esp. Oceanogr. 17: 107-120.

San Vicente C., Sorbe J.-C. 2013. Comparative life-histories, population dynamics and productivity of Schistomysis populations (Crustacea, Mysida) in European shelf environments. J. Sea Res. 81: 13-32. https://doi.org/10.1016/j.seares.2013.03.009

Sars G.O. 1899. An account of the Crustacea of Norway. Vol. III: Cumacea. Part I \& II: Cumidae, Lampropidae (part.). Bergen Museum, Christiania $115 \mathrm{pp}$

Shalla S.H., Bishop J.D.D. 2007. Lampropidae (Crustacea: Cumacea) from the deep north-east Atlantic and the North Sea, with 
two new species of Hemilamprops and Mesolamprops. J. Mar. Biol. Ass. U.K. 87: 1191-1200.

https://doi.org/10.1017/S0025315407055063

Sorbe J.C. 1981. Rôle du benthos dans le régime alimentaire des poissons démersaux du secteur sud-Gascogne. Kieler Meeresforsch., Sonderh. 5: 479-489.

Sorbe J.C. 1984. Contribution à la connaissance des peuplements suprabenthiques néritiques sud-Gascogne. Thèse d'Etat, Université de Bordeaux 1.

Sorbe J.C. 1999. Deep-sea macrofaunal assemblages within the Benthic Boundary Layer of the Cap-Ferret Canyon (Bay of Biscay, NE Atlantic). Deep-Sea Res. II 46: 2309-2329. https://doi.org/10.1016/S0967-0645(99)00064-8

Sorbe J.C, Elizalde M. 2014. Temporal changes in the structure of a slope suprabenthic community from the Bay of Biscay (NE Atlantic Ocean). Deep-Sea Res. II 106: 179-191. https://doi.org/10.1016/j.dsr2.2013.09.041

Sorbe J.C., Weber O. 1995. Influence de la profondeur et des sédiments superficiels sur la structure des communautés suprabenthiques bathyales sud-Gascogne. Actas del IV Coloquio Internacional sobre Oceanografía del Golfo de Vizcaya, pp. 183-194.

Stanley D.J., Addy S.K., Behrens E.W. 1983. The mudline: variability of its position relative to shelfbreak. In: Stanley D.J., Moore G.T., et al. (eds). The Shelfbreak: Critical Interface on Continental Margins, Special Publications of SEPM. https://doi.org/10.2110/pec.83.06.0279

Vaquer-Sunyer R., Duarte C.M. 2008. Thresholds of hypoxia for marine biodiversity. Proc. Natl. Acad. Sci. U.S.A. 105: 15452-15457.

https://doi.org/10.1073/pnas.0803833105

Watling L., Gerken S. 2019. World Cumacea Database. World Register of Marine Species. Cumacea. Accessed on 2019-02-03 through:

ht t p://ww w. marinespecies. org/aphia. php? $=$ taxdetails $\&$ id $=1137$

\section{SUPPLEMENTARY MATERIAL}

The following supplementary material is available through the online version of this article and at the following link:

http://scimar.icm.csic.es/scimar/supplm/sm05031esm.pdf

Table S1 - Vertical distribution of cumacean individuals in the near-bottom water layers $(\mathrm{N} 1,10-40 \mathrm{~cm} ; \mathrm{N} 2,45-75 \mathrm{~cm} ; \mathrm{N} 3$, 80-110 cm; N4, 115-145 cm above the seafloor) sampled by a suprabenthic sledge at 13 stations on the southern margin of the Cap Ferret Canyon. TS09 samples only partially studied due to bad conservation of material.,$- 0 ; *$, damaged specimens. 


\section{Bathyal cumacean assemblages from the southern margin of the Cap Ferret Canyon (SE Bay of Biscay)}

Jordi Corbera, Jean Claude Sorbe

Supplementary material 
S2 • J. Corbera and J.C. Sorbe

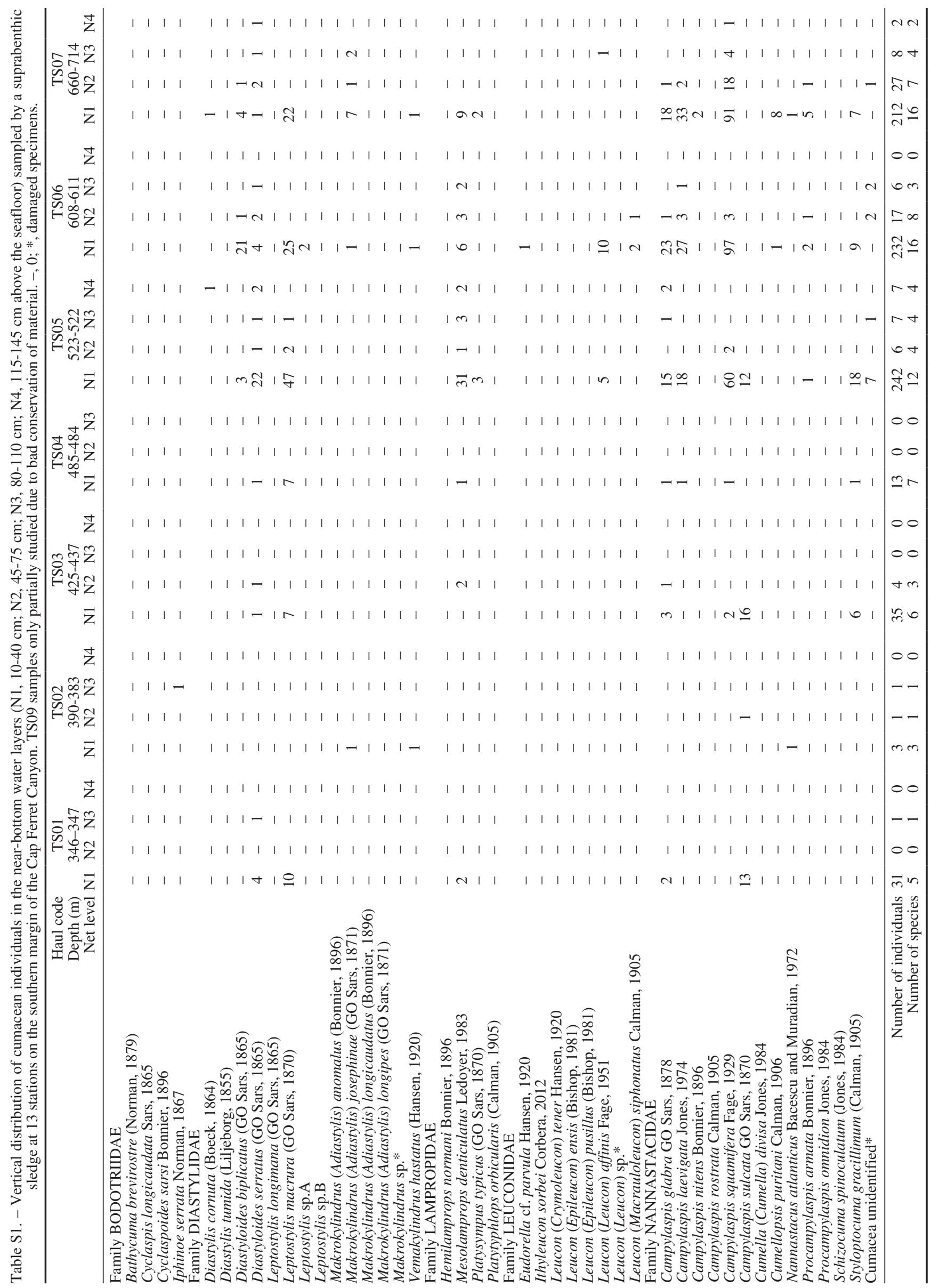




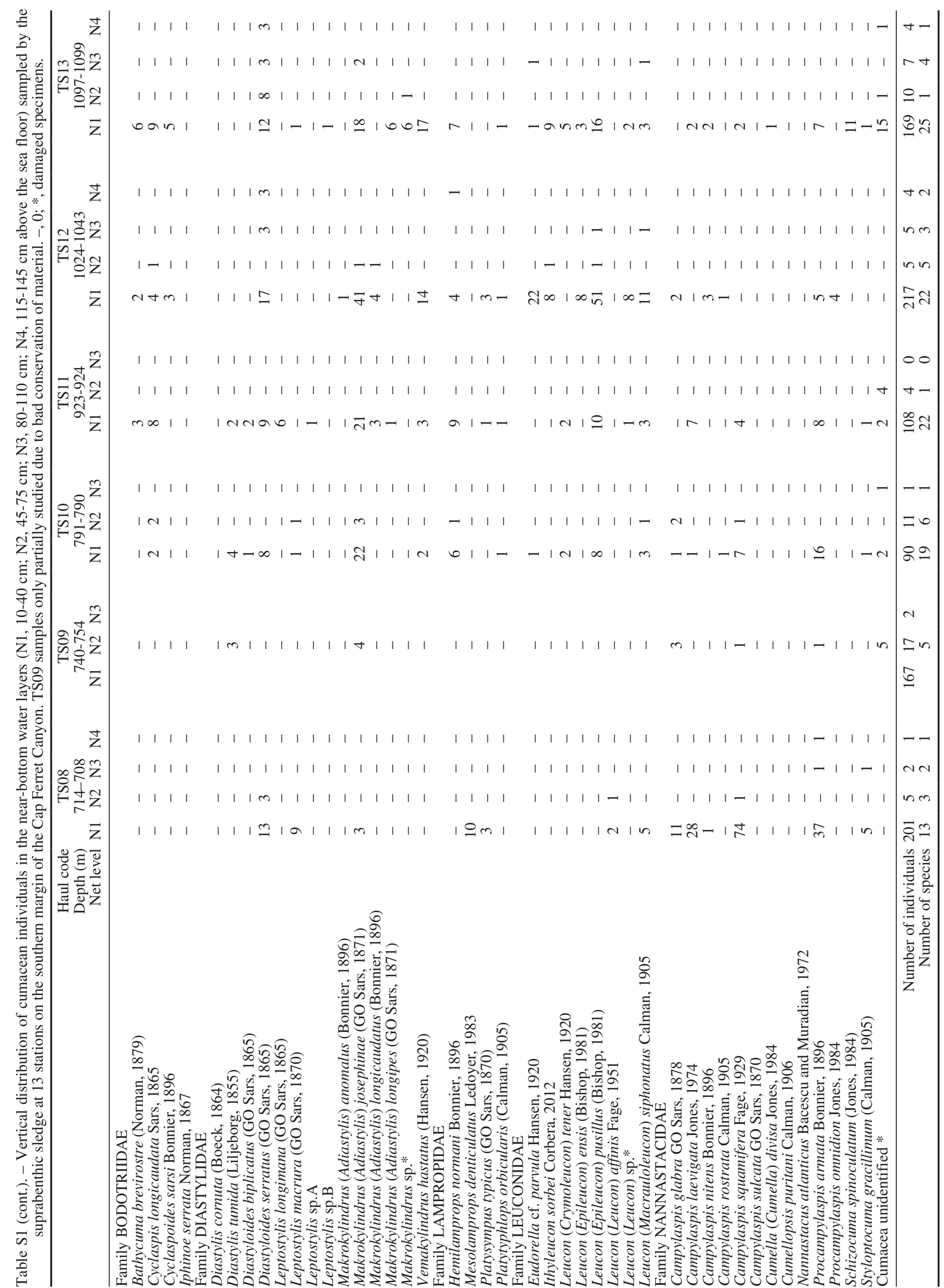

